\title{
Systems toxicology assessment of the biological impact of a candidate Modified Risk Tobacco Product on human organotypic oral epithelial cultures
}

Filippo Zanetti ${ }^{1}$, Alain Sewer ${ }^{1}$, Carole Mathis ${ }^{1}$, Anita R. Iskandar ${ }^{1}$, Radina Kostadinova ${ }^{1}$, Walter K. Schlage ${ }^{2}$, Patrice Leroy ${ }^{1}$, Shoaib Majeed ${ }^{1}$, Emmanuel Guedj ${ }^{1}$, Keyur Trivedi ${ }^{1}$, Florian Martin ${ }^{1}$, Ashraf Elamin ${ }^{1}$, Céline Merg ${ }^{1}$, Nikolai V. Ivanov ${ }^{1}$, Stefan Frentzel ${ }^{1}$, Manuel C. Peitsch ${ }^{1}$, and Julia Hoeng ${ }^{1}$

${ }^{1}$ Philip Morris International Research and Development, Quai Jeanrenaud 5, 2000 Neuchâtel, Switzerland

${ }^{2}$ Biology Consultant, Max-Baermann-Str. 21, 51429 Bergisch Gladbach, Germany 


\section{Supplementary Material}

\section{Supplementary Materials and Methods}

RNA and miRNA purification

Total RNA including microRNA (miRNA) was isolated after washing the cultures twice with cold $\left(4{ }^{\circ} \mathrm{C}\right) \mathrm{PBS}$ at both the basal and apical sides. The cells were then disrupted in $700 \mu \mathrm{L}$ QIAzol $^{\mathrm{TM}}$ lysis buffer (Qiagen, Hilden, Germany) followed by RNA extraction using a Qiagen miRNeasy Mini Kit and a QIAcube robot (Qiagen). The quantity of purified RNA was determined using a NanoDrop ${ }^{\mathrm{TM}}$ ND8000 spectrophotometer (Thermo Fisher Scientific, Waltham, MA, USA), while the quality of the RNA was analyzed using an Agilent 2100 Bioanalyzer (Agilent Technologies) considering only RIN values of 6 and above.

\section{mRNA microarray}

The Affymetrix HT 3' IVT PLUS kit (Affymetrix, Santa Clara, CA, USA) was used to reversetranscribe $100 \mathrm{ng}$ total RNA into cDNA, which was then labeled, amplified to cRNA (complementary RNA) and fragmented. The cRNA concentration in the final hybridization cocktail $(50.1 \mathrm{ng} / \mu \mathrm{L})$ was hybridized onto a GeneChip ${ }^{\circledR}$ Human Genome U133 Plus 2.0 Array (Affymetrix). Arrays were washed and stained on a GeneChip ${ }^{\circledR}$ Fluidics Station FS450 DX (Affymetrix) using the supplier's protocol FS450_0001 and scanned using a GeneChip ${ }^{\circledR}$ Scanner 3000 7G (Affymetrix). The AGCC software automatically gridded the DAT file image and extracted probe cell intensities into a CEL file.

\section{Processing raw CEL files of the mRNA microarray}

CEL files were background-corrected, normalized and summarized using frozen-Robust Microarray Analysis (fRMA). ${ }^{1}$ Background correction and quantile normalization was used to generate microarray expression values from all arrays passing quality control checks, which was performed using the custom CDF environment HGU133Plus2_Hs_ENTREZG v16.0. ${ }^{2}$ A logintensities plot, normalized-unscaled standard error plot (NUSE), relative log expression plot (RLE), median absolute value RLE (MARLE) and pseudo-images and raw images were generated using $\mathrm{R}$ packages for quality checks (AffyPLM). ${ }^{3,4} \mathrm{CEL}$ files fulfilling at least one of the following quality metric rules were dropped from further analysis: a) A pseudo-image 
displaying a spatial pattern covering approximately $10 \%$ of the pseudo-image; b) Median NUSE $>1.05$; c) $\mid$ Median RLE $>0.1$; and d) (MARLE-median(MARLE))|/ (1.4826*mad(MARLE)) $>1 / \sqrt{ } 0.01$, where mad is the median absolute deviation). Subsequently, the RLE and NUSE-based metrics were recomputed, until no more CEL files were removed. This led to a final mRNA expression matrix containing 18,604 rows (Entrez Gene IDs) and 325 columns (CEL files), which was subsequently submitted to the ArrayExpress repository (ID: E-MTAB-4742).

For each experimental factor combination, test item, concentration and post-exposure, a model for estimating the treatment effect was fitted with LIMMA, ${ }^{5}$ by including the covariate exposure run as a blocking variable, thereby accounting for the pairing during individual exposure runs (exposed versus the air control). The $p$-values for each computed effect were adjusted across genes using the Benjamini-Hochberg false discovery rate (FDR) method. ${ }^{6}$ Differentially expressed genes (DEGs) were defined as the set of genes whose FDR was $<0.05$.

\section{Calculating Network Perturbation Amplitude for the analysis of transcriptomics data}

The Network Perturbation Amplitude (NPA) methodology aims to quantify the biological response measured by a whole transcriptome investigation in terms of causal network models. ${ }^{7,8}$ The collection of causal biological networks models used in this study has been published in the human network suite CBN v1.3. ${ }^{9}$ The 28 relevant network models (for buccal epithelium) considered here are listed in Table 2. The networks range between a few dozen and two hundred nodes. The nodes of the networks correspond to biological entities (e.g., protein abundances, protein activities, chemical compounds, or biological processes, all described as molecular entities). Each of the edges in the network models carries a causal directionality between two nodes, based on information reported in the scientific literature. For many nodes of the network models, literature-derived information indicating that they regulate the expression levels of certain genes is also available.

The NPA algorithm is based on backward reasoning, by which changes in gene expression levels are considered to be the consequences of the activity changes of upstream network nodes. The node activity changes are determined by a fitting procedure that infers the values that best satisfy the directionality of the causal relationships (positive or negative signs) contained in the network model, while being constrained by the experimental gene differential expression data. ${ }^{8}$ The 
inferred activation (or inhibition) of the nodes and the causality relationships of the network edges permit the computation of the amplitude of the treatment-induced perturbation on the network as a whole. This quantity is accompanied by three statistics testing its uncertainty and its specificity with respect to the network structure, so that a network perturbation is considered as significant only when all three statistics are significant. ${ }^{8}$

\section{Calculating Biological Impact Factors for transcriptomics data}

The Biological Impact Factor (BIF) methodology provides high-level mechanistic insights in terms of the biological processes contained in the suite of 28 causal networks models listed in table 2. ${ }^{10,11}$ Briefly, this methodology enables the derivation of the global-level BIF, which measures the overall biological impact, and of the network family-level BIFs, which correspond to the four families regrouping the 29 networks models: cell fate (CFA), cell proliferation (CPR), cell stress (CST), and inflammation (IPN). The BIF calculations are based on the aggregation of NPA values via a between-treatment weighting scheme and a filtering procedure that only retains the significantly perturbed network perturbations, thereby enforcing the specificity of the BIF results.

\section{Gene-Set Analysis of transcriptomics data}

Gene-set analysis (GSA) of gene differential expressions was performed using the Piano package available in the $\mathrm{R}$ statistical environment. ${ }^{12} \mathrm{We}$ restricted GSA analysis to pathways obtained from the KEGG database ${ }^{13}$ and exported into $\mathrm{R}$ objects using the package graphite. ${ }^{14}$ For the GSA, the fold-change was used as the gene-level statistic and the mean was used as the gene-set enrichment score. Statistical significances of the gene sampling (Q1, first symbol on the heat map) and of the self-contained null hypothesis (Q2, second symbol on the heat map) were used to test the null hypothesis. ${ }^{15}$ For each test, the $p$-value was adjusted using the Benjamini-Hochberg procedure.

\section{miRNA microarray}

The FlashTagTM Biotin HSR kit (Affymetrix) was used to label miRNA. Total RNA (200 ng) containing low molecular weight RNA was subjected to a tailing reaction followed by ligation of 
the biotinylated signal molecule to the target RNA sample. The arrays (miRNA version 4.0) were incubated in the GeneChip ${ }^{\circledR}$ Hybridization Oven 645 (Affymetrix). Arrays were washed and stained on a GeneChip ${ }^{\circledR}$ Fluidics Station FS450 DX (Affymetrix) with supplier's protocol FS450_0002 and scanned using a GeneChip ${ }^{\circledR}$ Scanner 3000 7G (Affymetrix). The AGCC software automatically gridded the DAT file image and extracted probe cell intensities into a CEL file.

\section{Processing of raw CEL files of the miRNA microarray}

The CEL files were read using the Bioconductor oligo package for the $R$ statistical software environment. $^{16-18}$ The quality of the miRNA array data was controlled using the arrayQualityMetrics package ${ }^{19}$ and examined according to the following four metrics: a) the distances between arrays at the raw data level; b) the distances between arrays at the normalized data level; c) the Normalized Unscaled Standard Errors (NUSE); and d) the array intensity distributions. Arrays that were found to be outliers in at least two of the metrics were discarded. The quality criteria were then iteratively re-examined on the remaining arrays until all were accepted. The normalized probe-level data were obtained by applying robust multi-array (RMA) normalization and summarized using the median polish method at probeset-level., 20

Using the Affymetrix annotation and the latest miRBase miRNA nomenclature (released on 21 June 2014), ${ }^{21}$ only the probesets pertaining to the human species were kept. According to Affymetrix instructions, only the miRNA probesets with significantly higher intensity values than their matched background probes (based on the GC content and sequence length) were considered to be "detected" (see the "Detection" section in Affymetrix manual). ${ }^{22}$ A $p$-value threshold of 0.01 determined the detection calls based on Wilcoxon tests. If a miRNA probeset was detected in more than $50 \%$ of the samples in at least one sample group, then it was kept for further analysis; otherwise, it was discarded. To keep the current buccal results compatible with those from a similar study using nasal cultures, ${ }^{23}$ we further restricted our expression matrix to human miRNA probesets that are present on both arrays, versions 3.0 and 4.0. 
For each comparison, a submatrix was extracted from the global expression matrix by keeping only the samples belonging to the corresponding treatment or control groups, as well as the miRNA probesets that were detected in more than $50 \%$ of the samples in at least one of these two groups. As was done for mRNAs, linear models for differential expressions were applied on all resulting submatrices using the moderated $t$ statistics implemented in the limma package. ${ }^{24}$ The models included an additional variable taking into account the exposure runs, which improved the fitting procedure in limma. Subsequently, adjusted $p$-values were obtained following multiple testing corrections using the Benjamini-Hochberg FDR method. The miRNAs below the FDR threshold of 0.05 were considered differentially expressed.

\section{miRNA functional analysis}

For the biological interpretation of the miRNA data, the list of differentially expressed miRNAs was further condensed by considering those that have a fold-change of at least 0.5. Subsequently, the selected miRNAs were clustered using Euclidean distance, resulting in ten different miRNA clusters. The selected miRNAs were matched to their mRNA targets (experimentally established or predicted) according to Diana-TarBase version 7.0. Furthermore, we selected only the mRNA targets that were also present in our transcriptome dataset. For a given miRNA cluster, only the mRNAs inversely correlated with the miRNAs with a coefficient correlation of -0.5 were considered. (Pearson correlation was calculated for the mean differentially expressed miRNA in a given cluster and the corresponding genes extracted from TarBase). This approach selected fewer than 50 genes per miRNA cluster. 


\section{Supplementary Discussion: differential expression of genes related to the observed histological changes}

In support of the histological findings we analyzed the transcriptional levels of a series of genes involved in keratinization and adhesion, as well as barrier formation. We observed that the majority of cytokeratin genes were downregulated by 3R4F CS. Some considerations must accompany this result: (1) Because RNA is extracted from the full thickness epithelium, basal-tosuprabasal expression shifts for certain cytokeratins, for example, the supra-basal expression of KRT14 in proliferative lesions, could remain undetected if there were no net change in expression of the molecule of interest. (2) More important, it was demonstrated for several cytokeratins that their upregulation during stress was predominantly at the post-transcriptional level. That is, longlived mRNA from cytokeratins found in keratinizing lesions remained untranslated under normal conditions, allowing for a rapid adaptive response to mechanical (mastication) or chemical stress. This was shown by comparing immunohistochemistry (IHC) and fluorescent in situ hybridization (FISH) staining for KRT1 and KRT10 in normal buccal epithelium. Basal cells did not contain the proteins but expressed the mRNAs and, in supra-basal cells, both proteins and mRNA were present. $^{25,26}$ Also, the expression of KRT19 mRNA was observed in cells that were more basal and supra-basal than those expressing KRT19 protein. ${ }^{27}$ Another possibility is that, for KRT1 and KRT 10, post-transcriptional upregulation of their translation could be involved. That is, preexisting mRNA might have been depleted faster than it could be replenished by transcription, leading to a net decrease in mRNA abundance. However, such a proposed mechanism would need to be confirmed by measurements of the respective KRT proteins, that is, by IHC. This may also be the case for KRT19 mRNA-expressing (basal or Merkel) cells, which showed an initial decrease in mRNA followed by an increase. The basal cell associated KRT5, KRT14 and KRT15 mRNAs were also decreased, while expression of ITGA6, a key constituent of hemi-desmosomes, was increased at $72 \mathrm{~h}$ after an initial decrease, similar to the findings with KRT19. ITGA6 was reportedly increased in inflammatory epithelia. ${ }^{28-30}$ Though the occurrence of Merkel cells was not reported in reconstituted buccal epithelium, the coincident late increases in KRT7, KRT19, CHGA and S100A1 would support such an effect, though decreased MUC1 expression would support the opposite conclusion. Again, a confirmatory IHC analysis could elucidate the cellular basis of these changes in differentiation. Regarding proliferative changes, the opposite expression patterns of MKI67 and PCNA and the weak and transient KRT16 increase failed to provide 
support. The prominent increase in KRT17 expression may have indicated a differentiation process related to the in vitro reconstitution because this was also observed in hetero-grafted in vitro epithelia. ${ }^{31}$ Given the previously reported lack of DEFB1 stimulation in response to bacterial infection, ${ }^{32}$ the $\mathrm{CS}$-induced increase might reflect a nonspecific response to irritation. In CS-exposed cultures, we observed increased expression of many genes involved in TJ structure and regulation. TJs play an important role in epidermal barrier formation and depletion of the CLDN1 gene in mice led to hyperkeratosis. ${ }^{33-35}$ Interestingly, in our dataset, CLDN1 transcripts were strongly downregulated, though other claudins were either not affected or were even upregulated by CS exposure. This result could be in accordance with observations by Furuse et al., that $C L D N^{-/}$mice, compared with wildtypes, did not show changes in expression of other claudins (e.g. CLDN4). ${ }^{35}$ Many other genes involved in TJ formation were upregulated, indicating a general adaptive response to CS-induced stress. Moreover, a loss of tight junctional $C L D N 1$, together with $Z O-1$, was observed in inflamed buccal mucosa following chemotherapy. ${ }^{36}$ We could interpret our findings as suggesting a rapidly initiated adaptive response to rescue or increase tight junctional barrier function. This is also supported by the increase in JAM1 (here denoted F11R) along with a weak JAM2 and JAM3 upregulation, effects consistent with an increase in tight junction components

In agreement with the histological findings, only at the highest concentration (1.09) did THS2.2 aerosol exposure produce some of the mRNA changes that were also observed with 3R4F CS exposure. However, these effects were of a lower magnitude and some of the mRNAs increased by CS were unchanged with THS2.2. There was no indication of any unique effects caused by THS2.2 exposure.

We observed that CS exposure induced a pronounced decrease in E-cadherin staining. Consistent with this, the mRNA for $C D H 1$, the gene encoding for E-cadherin, was strongly downregulated in $3 \mathrm{R} 4 \mathrm{~F}$ CS-exposed cultures. Other genes belonging to the cadherin family, like $\mathrm{CDH} 3$ and $\mathrm{CDH} 4$, were differently regulated, with a tendency to increase their expression at the later timepoints, probably as a compensatory reaction to the loss of E-cadherin.

In murine oral epithelium, desmoglein-1 (DSG1) and desmoglein-3 (DSG3) are the most abundant of the desmosomal cadherins, with DSG3 considered the typical mucosal isoform. ${ }^{37} \mathrm{We}$ found that expression of $D S G 1 / 2 / 3$ and some important desmosomal cadherins $(D S C 1 / 2 / 3)$ were 
strongly downregulated after 3R4F CS exposure. Similarly, DSG2 and DSG3 expression were consistently decreased in oral biopsies from smokers. ${ }^{38}$

The activation of the Notch pathway relies on cell-cell contact. ${ }^{39}$ The Notch signaling pathway is known to promote cellular adhesion, though the associated molecular mechanisms are still poorly understood. $^{39-41}$ We observed a significant downregulation in expression of NOTCH1/2 after 3R4F CS exposure. Conversely, we observed a strong upregulation of $D L L 1$, a gene encoding for Delta-like ligand 1. DLL1 is a membrane protein that activates signaling cascades by binding Notch in adjacent cells. ${ }^{42}$ The upregulation of DLL1 might represent a mechanism for the cell to maintain signaling in response to the downregulation of its ligand (NOTCH1). In histological sections, none of these alterations were observed in THS2.2 aerosol-exposed organotypic cultures. 


\section{References}

(1) McCall, M. N., Bolstad, B. M., and Irizarry, R. A. (2010) Frozen robust multiarray analysis (fRMA). Biostatistics 11, 242-253.

(2) Dai, M., Wang, P., Boyd, A. D., Kostov, G., Athey, B., Jones, E. G., Bunney, W. E., Myers, R. M., Speed, T. P., Akil, H., Watson, S. J., and Meng, F. (2005) Evolving gene/transcript definitions significantly alter the interpretation of GeneChip data. Nucleic Acids Res. 33, e175.

(3) Bolstad, B. M., Irizarry, R. A., Astrand, M., and Speed, T. P. (2003) A comparison of normalization methods for high density oligonucleotide array data based on variance and bias. Bioinformatics 19, 185-193.

(4) Gautier, L., Cope, L., Bolstad, B. M., and Irizarry, R. A. (2004) affy--analysis of Affymetrix GeneChip data at the probe level. Bioinformatics 20, 307-315.

(5) Smyth, G. K. (2004) Linear models and empirical bayes methods for assessing differential expression in microarray experiments. Stat. Appl. Genet. Mol. Biol. 3, Article3.

(6) Benjamini, Y., and Hochberg, Y. (1995) Controlling the False Discovery Rate: A Practical and Powerful Approach to Multiple Testing. J. R. Stat. Soc. Series B Stat. Methodol. 57, 289-300.

(7) Martin, F., Thomson, T. M., Sewer, A., Drubin, D. A., Mathis, C., Weisensee, D., Pratt, D., Hoeng, J., and Peitsch, M. C. (2012) Assessment of network perturbation amplitudes by applying high-throughput data to causal biological networks. BMC Syst. Biol. 6, 54.

(8) Martin, F., Sewer, A., Talikka, M., Xiang, Y., Hoeng, J., and Peitsch, M. C. (2014) Quantification of biological network perturbations for mechanistic insight and diagnostics using two-layer causal models. BMC Bioinformatics 15, 238.

(9) Boué, S., Talikka, M., Westra, J. W., Hayes, W., Di Fabio, A., Park, J., Schlage, W. K., Sewer, A., Fields, B., Ansari, S., Martin, F., Veljkovic, E., Kenney, R., Peitsch, M. C., and Hoeng, J. (2015) Causal biological network database: a comprehensive platform of causal biological network models focused on the pulmonary and vascular systems. Database 2015.

(10) Hoeng, J., Talikka, M., Martin, F., Sewer, A., Yang, X., Iskandar, A., Schlage, W. K., and Peitsch, M. C. (2014) Case study: the role of mechanistic network models in systems toxicology. Drug Discovery Today 19, 183-192.

(11) Thomson, T. M., Sewer, A., Martin, F., Belcastro, V., Frushour, B. P., Gebel, S., Park, J., Schlage, W. K., Talikka, M., and Vasilyev, D. M. (2013) Quantitative assessment of biological impact using transcriptomic data and mechanistic network models. Toxicol. Appl. Pharmacol. 272, 863-878.

(12) Varemo, L., Nielsen, J., and Nookaew, I. (2013) Enriching the gene set analysis of genome-wide data by incorporating directionality of gene expression and combining statistical hypotheses and methods. Nucleic Acids Res. 41, 4378-4391.

(13) Kanehisa, M., Goto, S., Sato, Y., Kawashima, M., Furumichi, M., and Tanabe, M. (2014) Data, information, knowledge and principle: back to metabolism in KEGG. Nucleic Acids Res. 42, D199-205.

(14) Sales, G., Calura, E., Cavalieri, D., and Romualdi, C. (2012) graphite - a Bioconductor package to convert pathway topology to gene network. BMC Bioinformatics 13, 20. 
(15) Ackermann, M., and Strimmer, K. (2009) A general modular framework for gene set enrichment analysis. BMC Bioinformatics 10, 47.

(16) Carvalho, B. S., and Irizarry, R. A. (2010) A framework for oligonucleotide microarray preprocessing. Bioinformatics 26, 2363-2367.

(17) Huber, W., Carey, V. J., Gentleman, R., Anders, S., Carlson, M., Carvalho, B. S., Bravo, H. C., Davis, S., Gatto, L., Girke, T., Gottardo, R., Hahne, F., Hansen, K. D., Irizarry, R. A., Lawrence, M., Love, M. I., MacDonald, J., Obenchain, V., Oles, A. K., Pages, H., Reyes, A., Shannon, P., Smyth, G. K., Tenenbaum, D., Waldron, L., and Morgan, M. (2015) Orchestrating high-throughput genomic analysis with Bioconductor. Nat. Methods $12,115-121$.

(18) R Core Team. (2013) R: A language and environment for statistical computing. R Foundation for Statistical Computing, Vienna, Austria. URL http://www.R-project.org/.

(19) Kauffmann, A., Gentleman, R., and Huber, W. (2009) arrayQualityMetrics--a bioconductor package for quality assessment of microarray data. Bioinformatics 25, 415416.

(20) Irizarry, R. A., Bolstad, B. M., Collin, F., Cope, L. M., Hobbs, B., and Speed, T. P. (2003) Summaries of Affymetrix GeneChip probe level data. Nucleic Acids Res. 31, e15.

(21) Kozomara, A., and Griffiths-Jones, S. (2013) miRBase: annotating high confidence microRNAs using deep sequencing data. Nucleic Acids Res. 42, D68-73.

(22) Affymetrix Inc. (2011) Affymetrix miRNA QCTool: User's Guide., In http://media.affymetrix.com/support/downloads/manuals/mirna_qctool_user_manual.pdf (Affymetrix, I., Ed.).

(23) Iskandar, A. R., Xiang, Y., Frentzel, S., Talikka, M., Leroy, P., Kuehn, D., Guedj, E., Martin, F., Mathis, C., Ivanov, N. V., Peitsch, M. C., and Hoeng, J. (2015) Impact Assessment of Cigarette Smoke Exposure on Organotypic Bronchial Epithelial Tissue Cultures: A Comparison of Mono-Culture and Coculture Model Containing Fibroblasts. Toxicol. Sci. 147, 207-221.

(24) Ritchie, M. E., Phipson, B., Wu, D., Hu, Y., Law, C. W., Shi, W., and Smyth, G. K. (2015) limma powers differential expression analyses for RNA-sequencing and microarray studies. Nucleic Acids Res. 43, e47.

(25) Bloor, B. K., Tidman, N., Leigh, I. M., Odell, E., Dogan, B., Wollina, U., Ghali, L., and Waseem, A. (2003) Expression of keratin K2e in cutaneous and oral lesions: association with keratinocyte activation, proliferation, and keratinization. Am. J. Pathol. 162, 963975.

(26) Bloor, B. K., Su, L., Shirlaw, P. J., and Morgan, P. R. (1998) Gene expression of differentiation-specific keratins (4/13 and 1/10) in normal human buccal mucosa. Lab. Invest. 78, 787-795.

(27) Su, L., Morgan, P. R., Thomas, J. A., and Lane, E. B. (1993) Expression of keratin 14 and $19 \mathrm{mRNA}$ and protein in normal oral epithelia, hairy leukoplakia, tongue biting and white sponge nevus. J. Oral Pathol. Med. 22, 183-189.

(28) Kose, O., Lalli, A., Kutulola, A. O., Odell, E. W., and Waseem, A. (2007) Changes in the expression of stem cell markers in oral lichen planus and hyperkeratotic lesions. J. Oral Sci. 49, 133-139.

(29) Mirowsk, G. W., Lozada-Nur, F., Dekker, N. P., MacPhail, L. A., and Regezi, J. A. (1996) Altered expression of epithelial integrins and extracellular matrix receptors in oral erythema multiforme. J. Cutan. Pathol. 23, 473-478. 
(30) Hormia, M., Virtanen, I., and Quaranta, V. (1992) Immunolocalization of integrin alpha 6 beta 4 in mouse junctional epithelium suggests an anchoring function to both the internal and the external basal lamina. J. Dent. Res. 71, 1503-1508.

(31) Yoshizawa, M., Koyama, T., Kojima, T., Kato, H., Ono, Y., and Saito, C. (2012) Keratinocytes of tissue-engineered human oral mucosa promote re-epithelialization after intraoral grafting in athymic mice. Journal of Oral and Maxillofacial Surgery 70, 11991214.

(32) Kimball, J. R., Nittayananta, W., Klausner, M., Chung, W. O., and Dale, B. A. (2006) Antimicrobial barrier of an in vitro oral epithelial model. Arch. Oral Biol. 51, 775-783.

(33) Morita, K., Miyachi, Y., and Furuse, M. (2011) Tight junctions in epidermis: from barrier to keratinization. Eur. J. Dermatol. 21, 12-17.

(34) Zhou, K., Muroyama, A., Underwood, J., Leylek, R., Ray, S., Soderling, S. H., and Lechler, T. (2013) Actin-related protein $2 / 3$ complex regulates tight junctions and terminal differentiation to promote epidermal barrier formation. Proc. Natl. Acad. Sci. U. S. A. 110, E3820-3829.

(35) Furuse, M., Hata, M., Furuse, K., Yoshida, Y., Haratake, A., Sugitani, Y., Noda, T., Kubo, A., and Tsukita, S. (2002) Claudin-based tight junctions are crucial for the mammalian epidermal barrier: a lesson from claudin-1-deficient mice. J. Cell Biol. 156, 1099-1111.

(36) Wardill, H. R., Logan, R. M., Bowen, J. M., Van Sebille, Y. Z., and Gibson, R. J. (2016) Tight junction defects are seen in the buccal mucosa of patients receiving standard dose chemotherapy for cancer. Supportive Care in Cancer 24, 1779-1788.

(37) Mahoney, M. G., Hu, Y., Brennan, D., Bazzi, H., Christiano, A. M., and Wahl, J. K., 3rd. (2006) Delineation of diversified desmoglein distribution in stratified squamous epithelia: implications in diseases. Exp. Dermatol. 15, 101-109.

(38) Donetti, E., Gualerzi, A., Bedoni, M., Volpari, T., Sciarabba, M., Tartaglia, G., and Sforza, C. (2010) Desmoglein 3 and keratin 10 expressions are reduced by chronic exposure to cigarette smoke in human keratinised oral mucosa explants. Arch. Oral Biol. $55,815-823$.

(39) Murata, A., and Hayashi, S. (2016) Notch-Mediated Cell Adhesion. Biology (Basel) 5.

(40) Murata, A., Yoshino, M., Hikosaka, M., Okuyama, K., Zhou, L., Sakano, S., Yagita, H., and Hayashi, S. (2014) An evolutionary-conserved function of mammalian notch family members as cell adhesion molecules. PLoS One 9, e108535.

(41) Watt, F. M., Estrach, S., and Ambler, C. A. (2008) Epidermal Notch signalling: differentiation, cancer and adhesion. Curr. Opin. Cell Biol. 20, 171-179.

(42) Lobry, C., Oh, P., Mansour, M. R., Look, A. T., and Aifantis, I. (2014) Notch signaling: switching an oncogene to a tumor suppressor. Blood 123, 2451-2459. 


\section{Supplementary Figures and Tables legends}

\section{Table S1. Sample Numbers.}

Table S2. Nicotine concentrations in the THS2.2 aerosol from various dilutions applied in the DRA phase. Nicotine concentrations are expressed as means \pm SEM. Abbreviations: N, number of samples (trapped in the EXtrelut ${ }^{\circledR} 3 \mathrm{NT}$ columns); SEM, standard error of the mean.

Table S3. Biological functions of the clustered miRNAs based on an enrichment analysis of their target genes using EnrichR. For each miRNA cluster displayed on Figure 9, the results for the "KEGG 2016" library available in the EnrichR webtool are shown for their anti-correlated potential targets (a more detailed explanation is given in the Material and Methods section).

Figure S1. Schematic representation of the Vitrocell ${ }^{\circledR}$ dilution and exposure chambers. Organotypic cultures were exposed to 3R4F CS or THS2.2 aerosols on the apical side simultaneously using two independent Vitrocell exposure systems, connected to different smoking machines (SM 2000-3R4F and SM 2000-THS2.2). The Vitrocell system has a dilution/distribution module where dilutions of 3R4F CS and THS2.2 aerosol were applied to reach the target nicotine concentrations in the CS or aerosol.

Figure S2. Mean carbonyl levels deposited in culture medium from diluted 3R4F CS and THS2.2 aerosol. Deposited carbonyls $(\mu \mathrm{g})$ were calculated per $\mathrm{mL}$ PBS during the $28 \mathrm{~min}$ exposures. $\mathrm{N}=8$.

Figure S3. Dose Range Finding experiment with 3R4F CS exposure. H\&E stained sections at $48 \mathrm{~h}$ post-exposure derived from 2 donors. 20× magnification. 3R4F CS dilution is reported as a percentage $(\%)$ on the left.

Figure S4. Morphological alterations following exposures, relative to air exposed controls. Values indicate the fold changes between the means for given features of each exposure condition, compared with air-exposed samples. Blue and red colors indicate negative or positive fold-changes, respectively. The mean difference between the contrasts that is below the raw $p$ value of 0.05 is marked with the symbol *.

Figure S5. Dose Range Assessment of THS2.2 aerosol. H\&E stained sections at $72 \mathrm{~h}$ postexposure. 20× magnification. Values in brackets represents the nicotine concentration applied $(\mathrm{mg} / \mathrm{L})(\mathbf{A})$. Mean cytotoxicity levels at $72 \mathrm{~h}$ post-exposure, determined using the AK assay, with values normalized to those of the positive control (Triton-X-treated samples) (B). Error bars indicate SEM. $\mathrm{N}=3$.

Figure S6. Individual profiles of secreted proinflammatory mediators following exposure. Mean concentrations of analytes measured in the basolateral medium of organotypic cultures at 24, 48 or 72 h post-exposure (PE) for each group. Error bars indicate SEM. N = 11-12. 
Figure S7. Heat map of the gene set analysis (GSA) results for the network-related gene set collection. The figure shows values of the gene set enrichment scores (see Material \& Methods). The overlaying asterisks “*” indicate statistical significance for Q1 (left-hand side) and Q2 statistics (right-hand side). The selection of the gene set collection was done based on matching between the network nodes and the gene sets.

Figure S8. Heatmap of the gene set analysis (GSA) results for the network-related gene set collection. The figure shows the same values and statistics as Supplementary Figure 7 and displaying only gene sets containing at least one statistically significant value. The content of the KEGG gene set collection is explained in Materials and Methods.

Figure S9. Impact of exposures on global gene expression and biological network models. Volcano plots representing differentially expressed genes (each dot represents a gene) for each exposure group as compared with the air control. The changes in gene expression ( $\mathrm{x}$-axis) are shown as increased and decreased when $>0$ and $<0$, respectively. Upregulated genes are represented in yellow, whereas downregulated are in blue. Statistical significance is displayed on the $\mathrm{y}$-axis (at FDR $p$-value $<0.05$ ).

Figure S10. Altered expression levels of genes regulating stress and inflammation after 3R4F and THS2.2 exposures.

The heatmap shows genes, related to different stress subnetworks (A) or inflammation (B), that were significantly altered, with significance of $0.05\left(^{*}\right)$ or $0.01(* *)$, in at least one contrast (exposed group vs. air control). The color gradient represents the alteration of mRNA levels ( $\log 2($ fold-change)). Gene symbols are listed on the left of the heatmap. The "*" indicate statistically significant gene differential expressions based on FDR values smaller than the 0.05 threshold, as explained in the Material and Methods section.

Figure S11. Impact of exposures on global miRNA expression and biological network models. Volcano plots representing the differentially expressed miRNA (each dot represents a gene) for each exposure group as compared with the air control. The changes in gene expression (x-axis) are shown as increased and decreased when $>0$ and $<0$, respectively. Upregulated genes are represented in yellow, whereas downregulated are in blue. Statistical significance is displayed on the $\mathrm{y}$-axis (at FDR $p$-value $<0.05$ ).

Figure S12. Correlation plots of the immunohistochemistry, cytotoxicity, CYP1A1/1B1 activity, and proinflammatory mediators across the experimental repetitions. The average of the differences of the endpoint values (exposed vs. control), which were obtained from the experimental repetition 1 and 2 (x-axis), were correlated to those obtained from the experimental repetition 3 and 4 (y-axis). Different symbols are used to highlight the different endpoints: AK (the normalized cytotoxicity measurement), CYP (the normalized activity of CYP1A1/1B1), and MAP (the logarithm based 10 of the concentration measured using Luminex based analyses of proinflammatory mediators). A 45-degree line is drawn to ease the visualization. A Spearman and Pearson correlations were computed.

Figure S13. Correlation plots of gene and miRNA fold-changes. Each dot represents a gene (A) or miRNA (B). The fold-change of a given gene or miRNA from the combined experimental 
repetitions 1 and 2 (x-axis; $\mathrm{N}=6$; with triplicates per experiment) was compared with the foldchange of the same gene or miRNA from the combined experiment 3 and 4 (y-axis; $N=6$; with triplicates per experiment). Abbreviations: Corr, correlation; Sp, Spearman correlation; DEG, differentially expressed gene. 


\section{Supplementary Tables and Figures}

\section{Table S1}

\begin{tabular}{|c|c|c|c|c|c|c|}
\hline 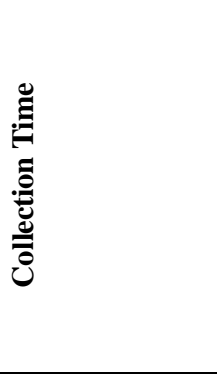 & ثิ: & 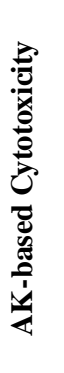 & 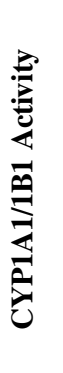 & 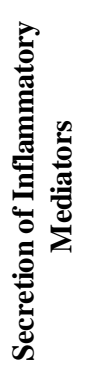 & 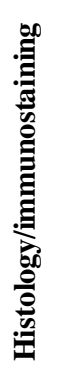 & 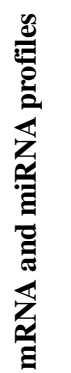 \\
\hline \multirow[t]{7}{*}{$4 \mathrm{~h} \mathrm{PE}$} & 3R4F (Air) & 13 & - & - & - & 12 \\
\hline & 3R4F (0.32) & 12 & - & - & - & 12 \\
\hline & 3R4F (0.51) & 12 & - & - & - & 12 \\
\hline & THS2.2 (Air) & 11 & - & - & - & 12 \\
\hline & THS2.2 (0.31) & 12 & - & - & - & 12 \\
\hline & THS2.2 (0.46) & 12 & - & - & - & 12 \\
\hline & THS2.2 (1.09) & 12 & - & - & - & 12 \\
\hline \multirow[t]{7}{*}{24 h PE } & 3R4F (Air) & 12 & 12 & 12 & - & 12 \\
\hline & 3R4F (0.32) & 12 & 12 & 12 & - & 12 \\
\hline & 3R4F (0.51) & 12 & 12 & 12 & - & 12 \\
\hline & THS2.2 (Air) & 12 & 12 & 12 & - & 12 \\
\hline & THS2.2(0.31) & 12 & 12 & 12 & - & 12 \\
\hline & THS2.2 (0.46) & 12 & 12 & 12 & - & 12 \\
\hline & THS2.2 (1.09) & 12 & 12 & 12 & - & 12 \\
\hline \multirow[t]{7}{*}{48 h PE } & 3R4F (Air) & 12 & 12 & 12 & 12 & 12 \\
\hline & 3R4F (0.32) & 12 & 12 & 12 & 12 & 12 \\
\hline & 3R4F $(0.51)$ & 12 & 12 & 12 & 12 & 12 \\
\hline & THS2.2 (Air) & 12 & 12 & 12 & 12 & 12 \\
\hline & THS2.2 (0.31) & 12 & 12 & 12 & 12 & 12 \\
\hline & THS2.2 (0.46) & 12 & 12 & 12 & 12 & 12 \\
\hline & THS2.2 (1.09) & 12 & 12 & 12 & 12 & 12 \\
\hline \multirow[t]{7}{*}{$72 \mathrm{~h} \mathrm{PE}$} & 3R4F (Air) & 12 & - & 12 & 12 & 12 \\
\hline & 3R4F (0.32) & 12 & - & 12 & 12 & 12 \\
\hline & 3R4F $(0.51)$ & 12 & - & 12 & 12 & 12 \\
\hline & THS2.2 (Air) & 12 & - & 12 & 12 & 12 \\
\hline & THS2.2 (0.31) & 12 & - & 12 & 12 & 12 \\
\hline & THS2.2 (0.46) & 12 & - & 12 & 12 & 12 \\
\hline & THS2.2 (1.09) & 12 & - & 12 & 12 & 12 \\
\hline \multirow[t]{5}{*}{72 h PE - DRA } & THS2.2 (Air) & 3 & - & - & 3 & - \\
\hline & THS2.2 (0.34) & 3 & - & - & 3 & - \\
\hline & THS2.2 (0.45) & 3 & - & - & 3 & - \\
\hline & THS2.2 (1.02) & 3 & - & - & 3 & - \\
\hline & THS2.2 (1.79) & 3 & - & - & 3 & - \\
\hline
\end{tabular}


Table S2

\begin{tabular}{|c|c|c|}
\hline Group & Aerosol concentration & $\begin{array}{c}\text { Average nicotine concentration } \\
\text { (mg/L) }\end{array}$ \\
\hline THS2.2 (0.31) & $25 \%$ & $0.336 \pm 0.014(\mathrm{~N}=3)$ \\
\hline THS2.2 Dose 2 (0.45) & $32 \%$ & $0.45 \pm 0.078(\mathrm{~N}=3)$ \\
\hline THS2.2 Dose 3 (1.02) & $80 \%$ & $1.024 \pm 0.256(\mathrm{~N}=3)$ \\
\hline THS2.2 Dose 4 (1.79) & $100 \%$ & $1.786 \pm 0.096(\mathrm{~N}=3)$ \\
\hline
\end{tabular}


Table S3

\begin{tabular}{|c|c|c|c|c|}
\hline Cluster & Overlap & $\begin{array}{c}\text { Adjusted. } \\
\text { P.value }\end{array}$ & Z.score & Genes \\
\hline \multicolumn{5}{|l|}{$\overline{\text { Cluster } \mathrm{A}}$} \\
\hline cholera infection & $2 / 41$ & 0.032824333 & -2.006087304 & ARF3;ADCY3 \\
\hline regulation of actin cytoskeleton & $2 / 201$ & 0.149291983 & -1.884997317 & ABI2;WASF2 \\
\hline \multicolumn{5}{|l|}{ Cluster B } \\
\hline pathogenic escherichia coli infection epec & $6 / 48$ & 0.003847188 & -2.034050355 & $\mathrm{CDH} 1 ; \mathrm{NCL} ; \mathrm{ABL} 1 ; \mathrm{CTNNB} 1 ; \mathrm{CLDN} 1 ; \mathrm{YWHAZ}$ \\
\hline pathogenic escherichia coli infection ehec & $6 / 48$ & 0.003847188 & -1.849593684 & CDH1;NCL;ABL1;CTNNB1;CLDN1;YWHAZ \\
\hline bladder cancer & $4 / 40$ & 0.086417171 & -1.861204975 & CDKN1A;CDH1;MDM2;FGFR3 \\
\hline cell cycle & $6 / 104$ & 0.086417171 & -1.700095607 & CDKN1A;CDKN2B;PRKDC;ABL1;MDM2;YWHAZ \\
\hline \multicolumn{5}{|l|}{ Cluster $\mathrm{C}$} \\
\hline huntingtons disease & $3 / 31$ & 0.01013905 & -2.028372048 & BAX;CALM2;TP53 \\
\hline glioma & $3 / 62$ & 0.032008463 & -2.055676806 & PIK3CB;CALM2;TP53 \\
\hline colorectal cancer & $3 / 84$ & 0.032008463 & -1.770253461 & BAX;PIK3CB;TP53 \\
\hline apoptosis & $3 / 81$ & 0.032008463 & -1.722318876 & BAX;PIK3CB;TP53 \\
\hline amyotrophic lateral sclerosis & $2 / 19$ & 0.032008463 & -1.512481168 & BAX;TP53 \\
\hline endometrial cancer & $2 / 52$ & 0.095620556 & -1.561869826 & PIK3CB;TP53 \\
\hline non small cell lung cancer & $2 / 53$ & 0.095620556 & -1.554762617 & PIK3CB;TP53 \\
\hline \multicolumn{5}{|l|}{ Cluster D } \\
\hline \multicolumn{5}{|l|}{ Cluster E } \\
\hline melanogenesis & $5 / 100$ & 0.431754732 & -1.936921748 & EDN1;ADCY9;TCF7;EP300;ADCY1 \\
\hline mapk signaling pathway & $6 / 248$ & 0.640389655 & -1.978608973 & PPM1A;CACNG8;RAPGEF2;MAPK14;CACNG4;MAP3K4 \\
\hline gnrh signaling pathway & $4 / 96$ & 0.640389655 & -1.919811757 & ADCY9;ADCY1;MAPK14;MAP3K4 \\
\hline adherens junction & $3 / 75$ & 0.640389655 & -1.723217892 & TCF7;EP300;WASF2 \\
\hline purine metabolism & $4 / 139$ & 0.640389655 & -1.560770935 & ADCY9;CANT1;ADK;ADCY1 \\
\hline neurodegenerative diseases & $2 / 38$ & 0.640389655 & -1.552667329 & ATN1;EP300 \\
\hline \multicolumn{5}{|l|}{ Cluster F } \\
\hline ubiquitin mediated proteolysis & $4 / 39$ & 0.002246096 & -1.976198212 & CDC20;ITCH;FBXW11;UBE2D1 \\
\hline hedgehog signaling pathway & $2 / 57$ & 0.419116675 & -1.859358248 & FBXW11;CSNK1D \\
\hline gap junction & $2 / 90$ & 0.419116675 & -1.661095294 & TUBAL3;CSNK1D \\
\hline \multicolumn{5}{|l|}{ Cluster G } \\
\hline adherens junction & $7 / 75$ & 0.050231954 & -1.857312822 & SMAD2;SMAD4;PTPRM;PTPN6;PVRL4;NLK;ACTG1 \\
\hline cell cycle & $8 / 104$ & 0.050231954 & -1.823998628 & SMAD2;GSK3B;SMAD4;TFDP1;MDM2;MCM4;BUB3;YWHAZ \\
\hline alzheimers disease & $3 / 28$ & 0.396515084 & -1.606235172 & APH1A;GSK3B;GAPDH \\
\hline \multicolumn{5}{|l|}{ Cluster H } \\
\hline ubiquitin mediated proteolysis & $1 / 39$ & 0.09222463 & -1.976198212 & CDC23 \\
\hline toll like receptor signaling pathway & $1 / 99$ & 0.09222463 & -1.84127121 & IFNAR1 \\
\hline natural killer cell mediated cy totoxicity & $1 / 129$ & 0.09222463 & -1.809465136 & IFNAR1 \\
\hline cell cycle & $1 / 104$ & 0.09222463 & -1.762047117 & CDC23 \\
\hline jak stat signaling pathway & $1 / 148$ & 0.09222463 & -1.716853786 & IFNAR1 \\
\hline cytokine cytokine receptor interaction & $1 / 247$ & 0.126218896 & -1.774863254 & IFNAR1 \\
\hline \multicolumn{5}{|l|}{ Cluster I } \\
\hline basal transcription factors & $1 / 33$ & 0.008945014 & -1.949170524 & TAF12 \\
\hline \multicolumn{5}{|l|}{ Cluster J } \\
\hline chronic myeloid leukemia & $4 / 75$ & 0.170383972 & -2.083794989 & CTBP1;AKT1;ACVR1B;HRAS \\
\hline endometrial cancer & $3 / 52$ & 0.170383972 & -1.995166802 & $\mathrm{CDH} 1 ; \mathrm{AKT} 1 ; \mathrm{HRAS}$ \\
\hline b cell receptor signaling pathway & $3 / 62$ & 0.170383972 & -1.754795161 & PPP3CA;AKT1;HRAS \\
\hline vegf signaling pathway & $3 / 68$ & 0.170383972 & -1.748644554 & PPP3CA;AKT1;HRAS \\
\hline natural killer cell mediated cytotoxicity & $4 / 129$ & 0.170383972 & -1.686365031 & PPP3CA;IFNGR2;HLA-C;HRAS \\
\hline melanoma & $3 / 71$ & 0.170383972 & -1.563068543 & CDH1;AKT1;HRAS \\
\hline
\end{tabular}




\section{Figure S1}

\section{Vitrocell Dilution and Exposure System}

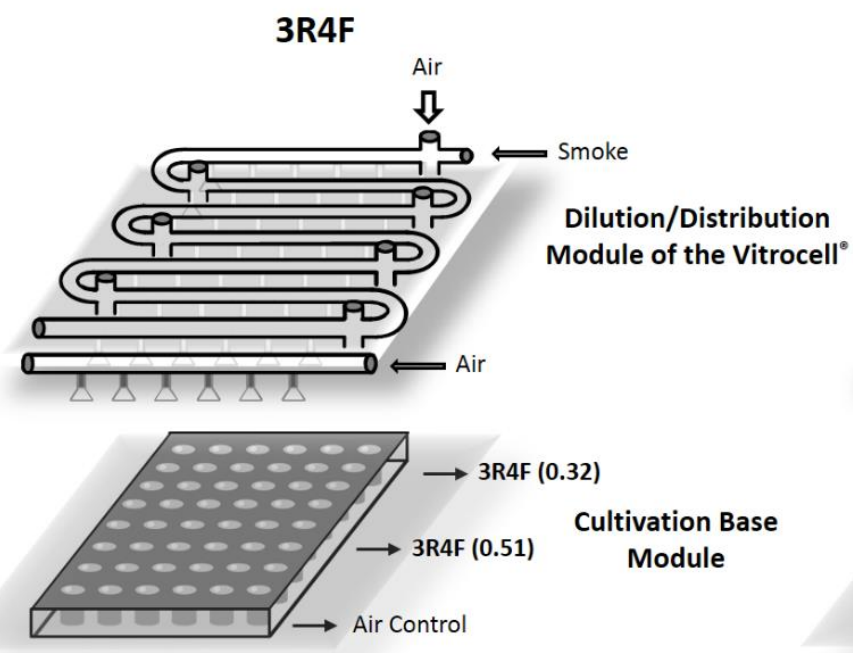

THS2.2

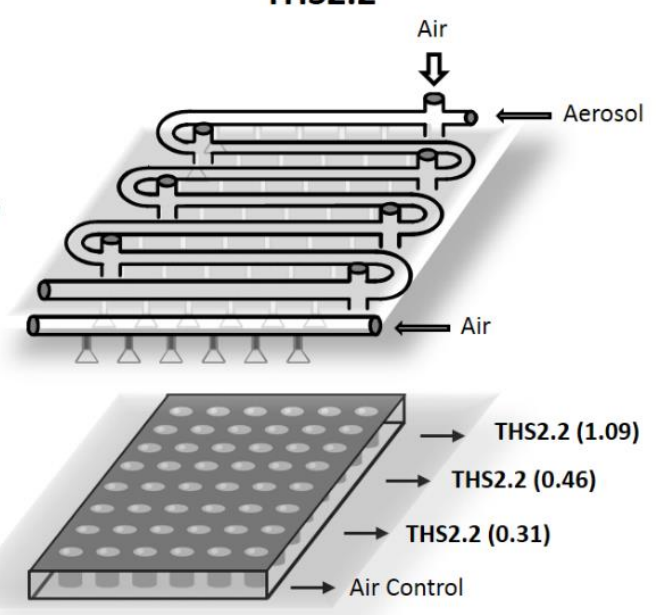




\section{Figure S2}
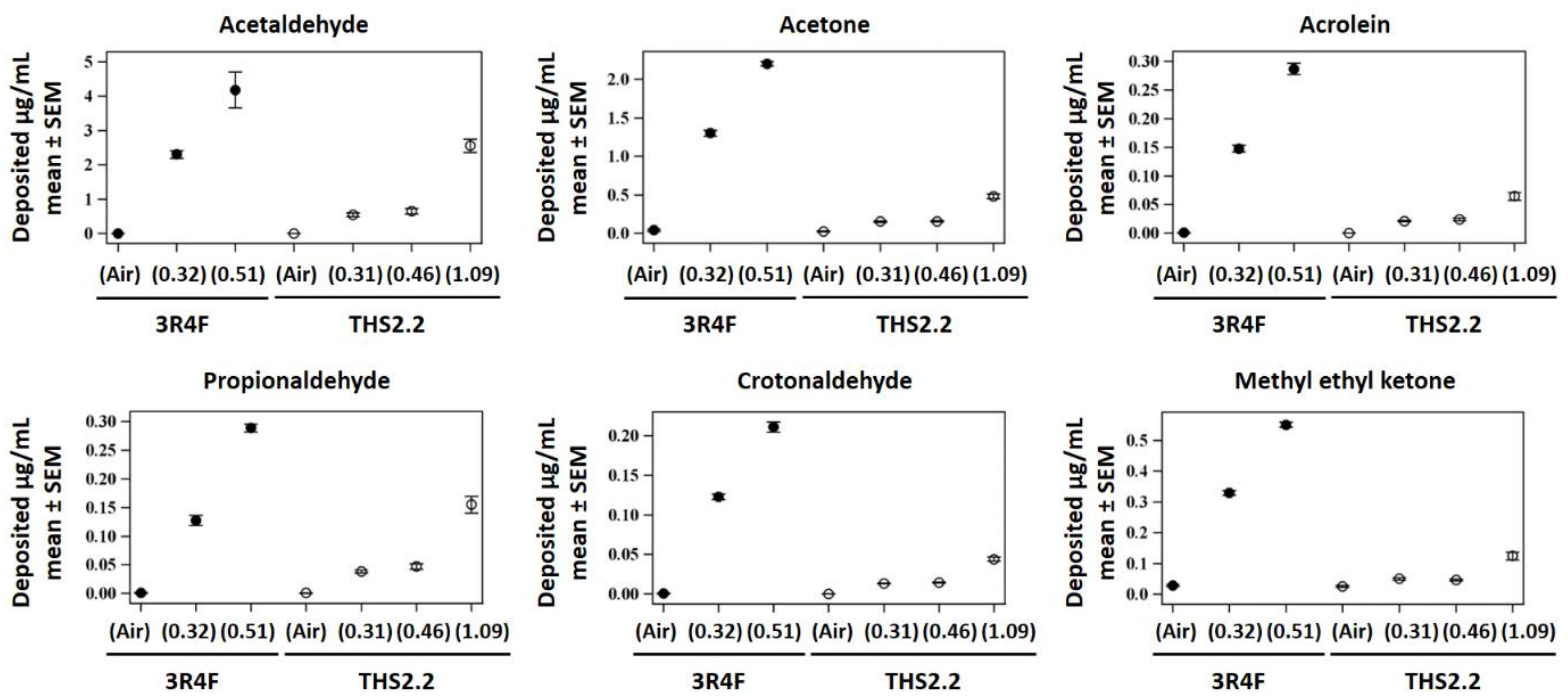


\section{Figure S3}

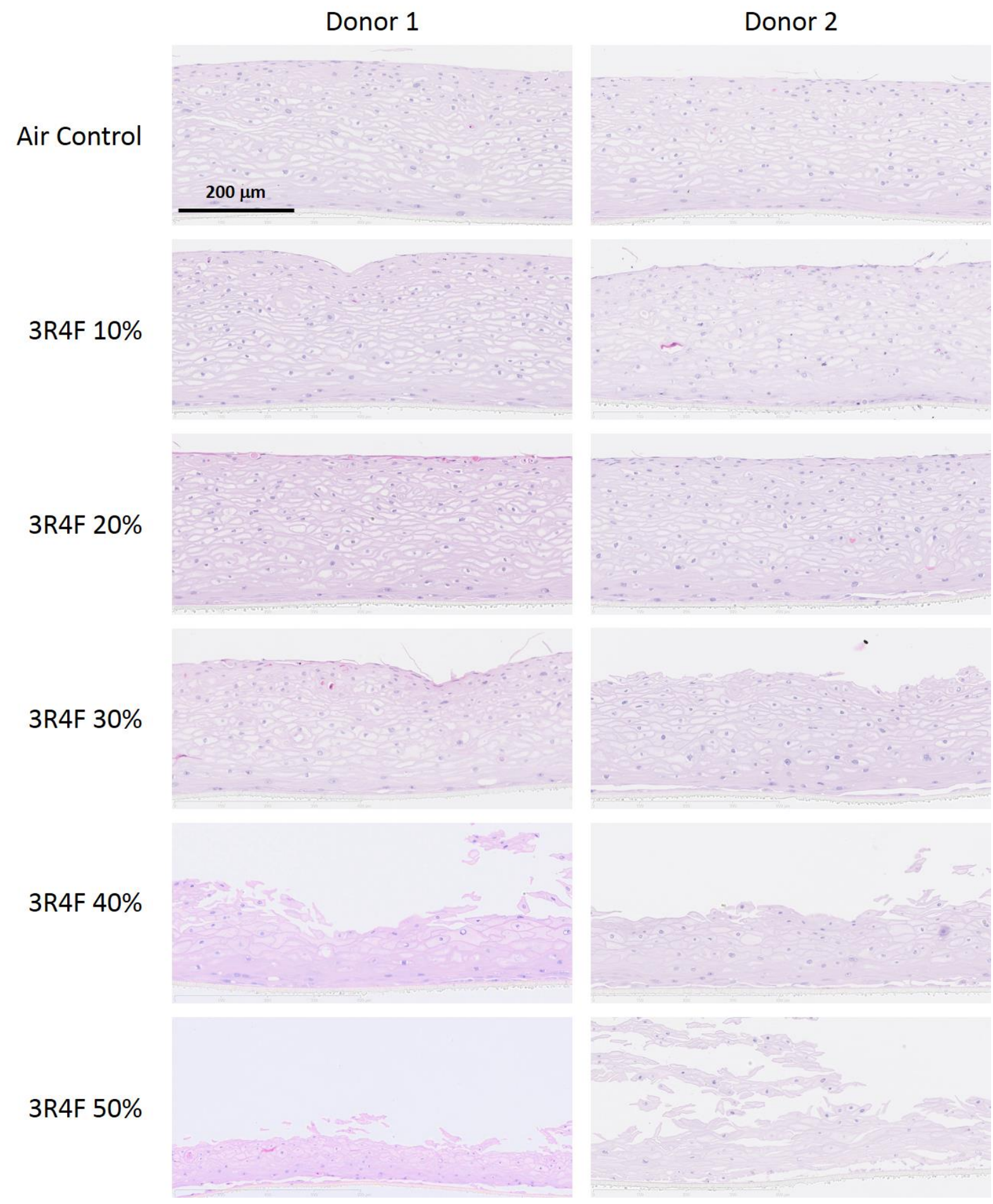




\section{Figure S4}

\begin{tabular}{|c|c|c|c|c|c|c|c|c|c|c|c|}
\hline Morphological findings & $48 \mathrm{~h}$ & $72 \mathrm{~h}$ & $48 \mathrm{~h}$ & $72 \mathrm{~h}$ & $48 \mathrm{~h}$ & $72 \mathrm{~h}$ & $48 \mathrm{~h}$ & $72 \mathrm{~h}$ & $48 \mathrm{~h}$ & $72 \mathrm{~h}$ & \multirow[t]{2}{*}{ Post-Exposure } \\
\hline Apical keratinization & 0.08 & 0.50 & 0.75 & $1.50^{*}$ & -0.17 & -0.17 & 0.25 & 0.00 & -0.17 & -0.33 & \\
\hline Cellular alteration & -0.33 & -0.33 & 0.25 & -0.33 & -0.25 & 0.00 & -0.25 & -0.25 & $-0.58^{*}$ & -0.25 & \multirow{3}{*}{1.58} \\
\hline Epithelial Atrophy & -0.17 & 0.42 & -0.17 & 0.00 & 0.42 & 0.08 & -0.08 & 0.00 & -0.08 & 0.00 & \\
\hline Cysts & 0.25 & $-0.58^{*}$ & -0.17 & $-0.75^{*}$ & 0.17 & -0.08 & 0.33 & -0.25 & 0.00 & -0.33 & \\
\hline Ectopic keratinization & 0.08 & $0.50^{*}$ & 0.08 & 0.42 & -0.25 & -0.08 & -0.17 & -0.17 & 0.00 & $-0.42^{*}$ & \multirow{5}{*}{ 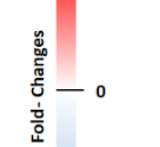 } \\
\hline Detachment of tissue below basal cell level & $0.75^{*}$ & 0.75 & $1.17^{*}$ & $1.58^{*}$ & 0.17 & 0.25 & -0.17 & 0.08 & 0.08 & 0.25 & \\
\hline Detachment of tissue above basal cell level & 0.50 & 0.25 & 0.00 & -0.42 & 0.17 & 0.08 & 0.33 & -0.08 & 0.25 & 0.08 & \\
\hline Extracellular granules & 0.00 & 0.00 & 0.00 & 0.00 & 0.00 & 0.00 & 0.00 & 0.00 & 0.00 & 0.00 & \\
\hline Cytoplasmic vacuolation & 0.08 & $-0.42^{*}$ & 0.25 & -0.42 & -0.17 & -0.08 & 0.33 & 0.00 & -0.42 & -0.33 & \\
\hline Increased desquamation & 0.33 & 0.67 & 0.17 & $1.50^{*}$ & 0.00 & 0.25 & 0.00 & $0.67 *$ & -0.17 & $0.67^{*}$ & \multirow{7}{*}{$\mathrm{Nic}(\mathrm{mg} / \mathrm{mL})$} \\
\hline Intracellular fine granular basophilic material & 0.25 & $0.50^{*}$ & $0.67^{*}$ & $1.00^{*}$ & 0.00 & 0.00 & 0.00 & 0.00 & 0.00 & 0.00 & \\
\hline Longitudinal cleft formation & 0.17 & $0.50^{*}$ & 0.25 & 0.42 & 0.17 & 0.17 & 0.17 & 0.25 & 0.00 & 0.25 & \\
\hline Single cell necrosis/ apoptosis & 0.00 & -0.08 & -0.17 & -0.25 & 0.00 & 0.17 & -0.33 & 0.00 & -0.17 & $-0.50^{*}$ & \\
\hline \multirow[t]{3}{*}{ Tissue thickness inhomogeneity } & -0.33 & -0.08 & $-0.42^{*}$ & -0.08 & 0.00 & 0.00 & -0.17 & 0.08 & 0.08 & 0.00 & \\
\hline & \multicolumn{2}{|c|}{ (0.32) vs (Air) } & \multicolumn{2}{|c|}{ (0.51) vs (Air) } & (0.31) & $s$ (Air) & \multicolumn{2}{|c|}{ (0.46) vs (Air) } & \multicolumn{2}{|c|}{ (1.09) vs (Air) } & \\
\hline & \multicolumn{4}{|c|}{ 3R4F } & \multicolumn{6}{|c|}{ THS2.2 } & \\
\hline
\end{tabular}




\section{Figure S5}

A

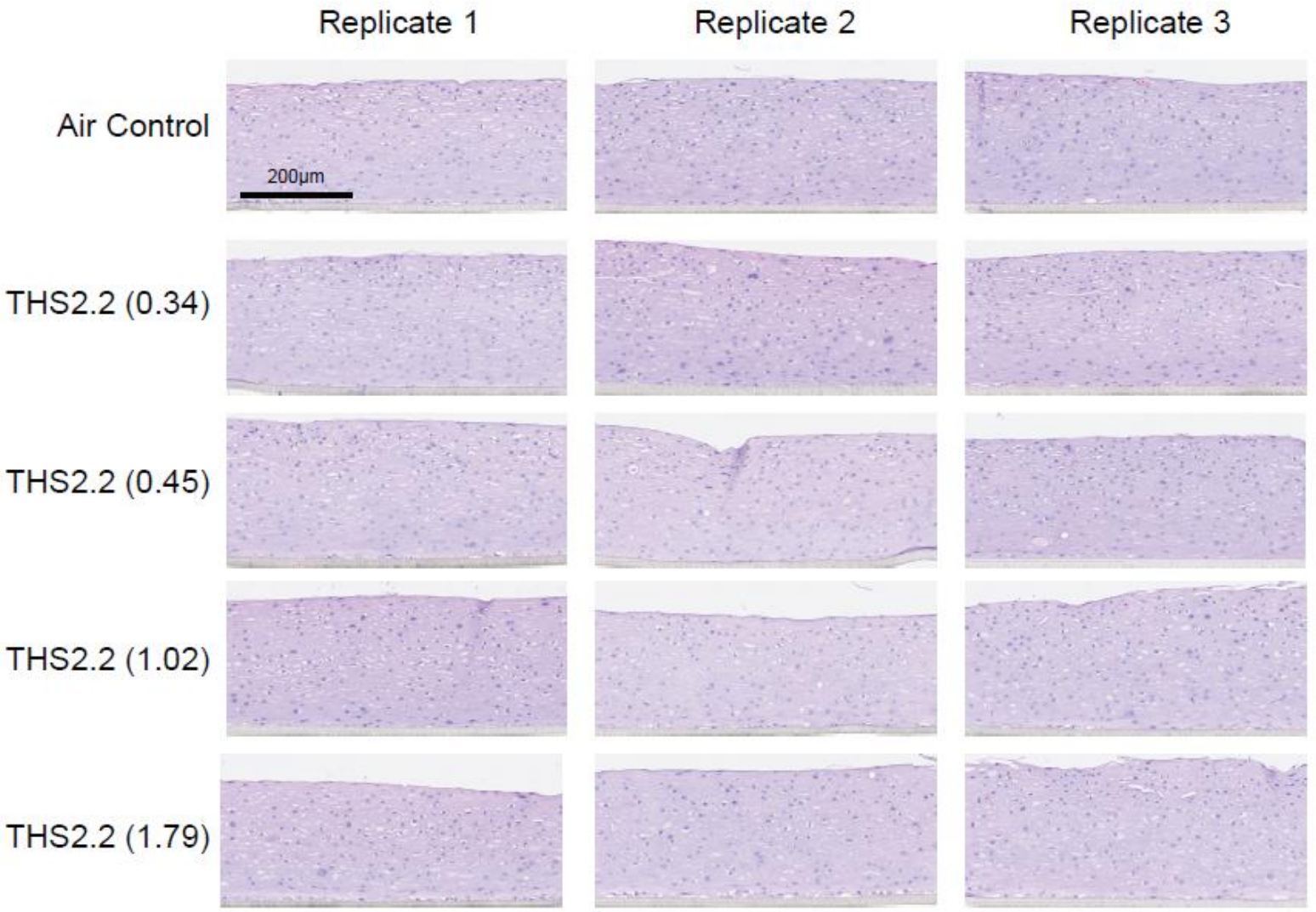

B

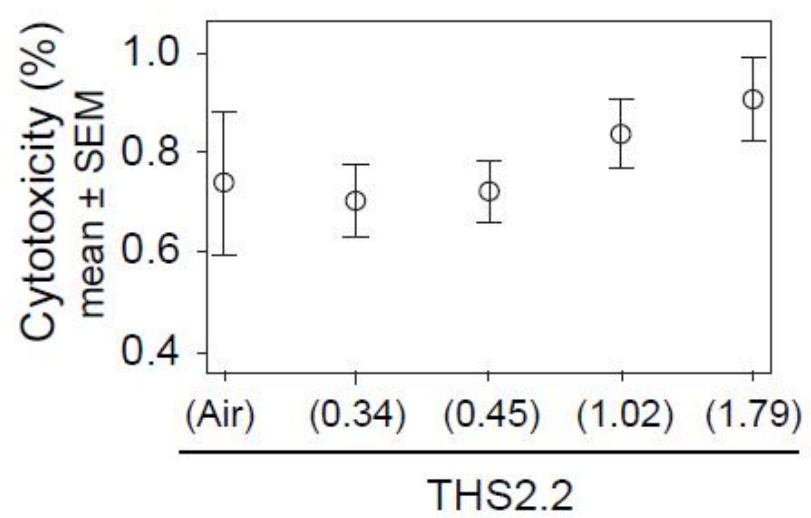




\section{Figure S6}
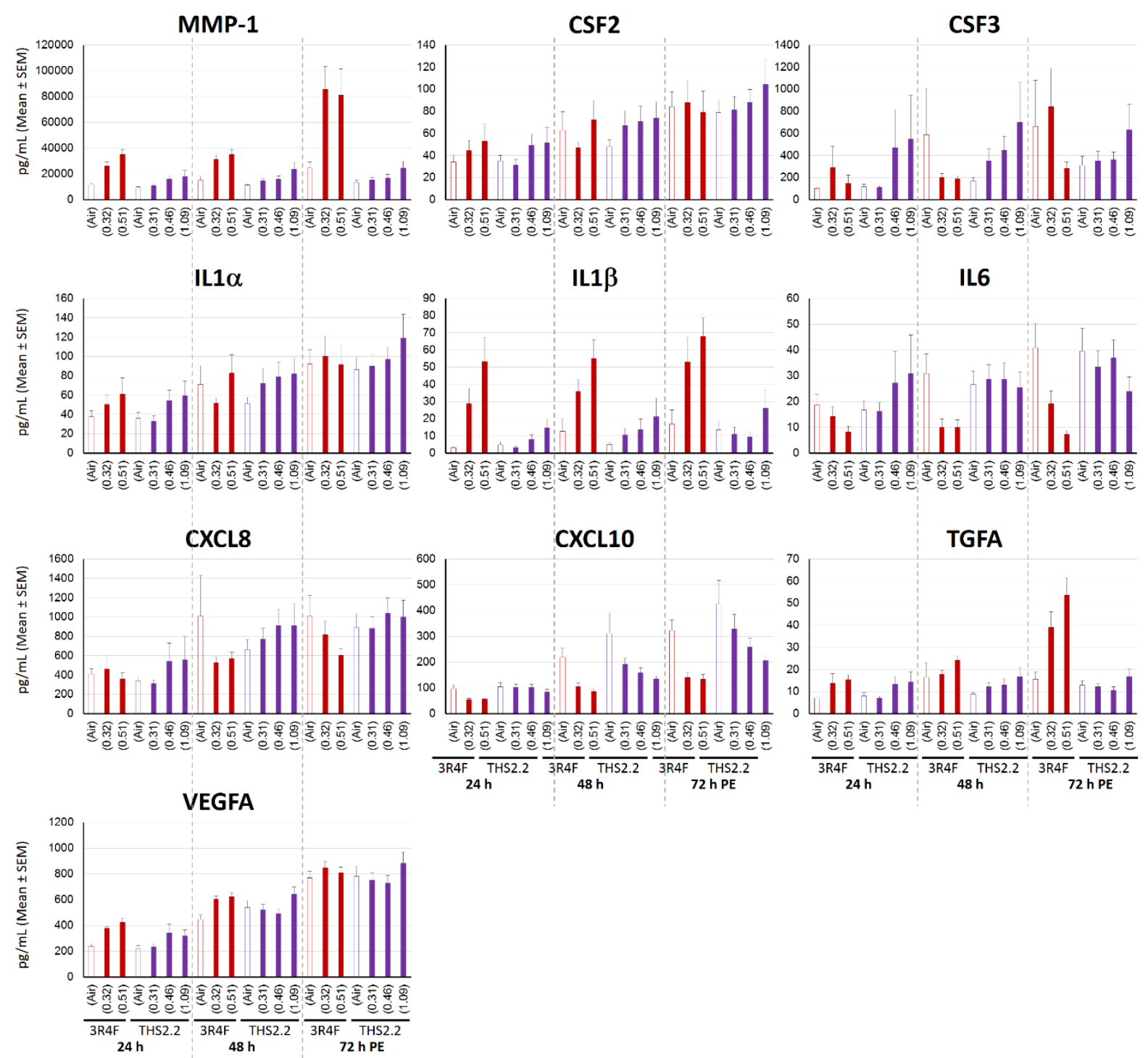
Figure S7

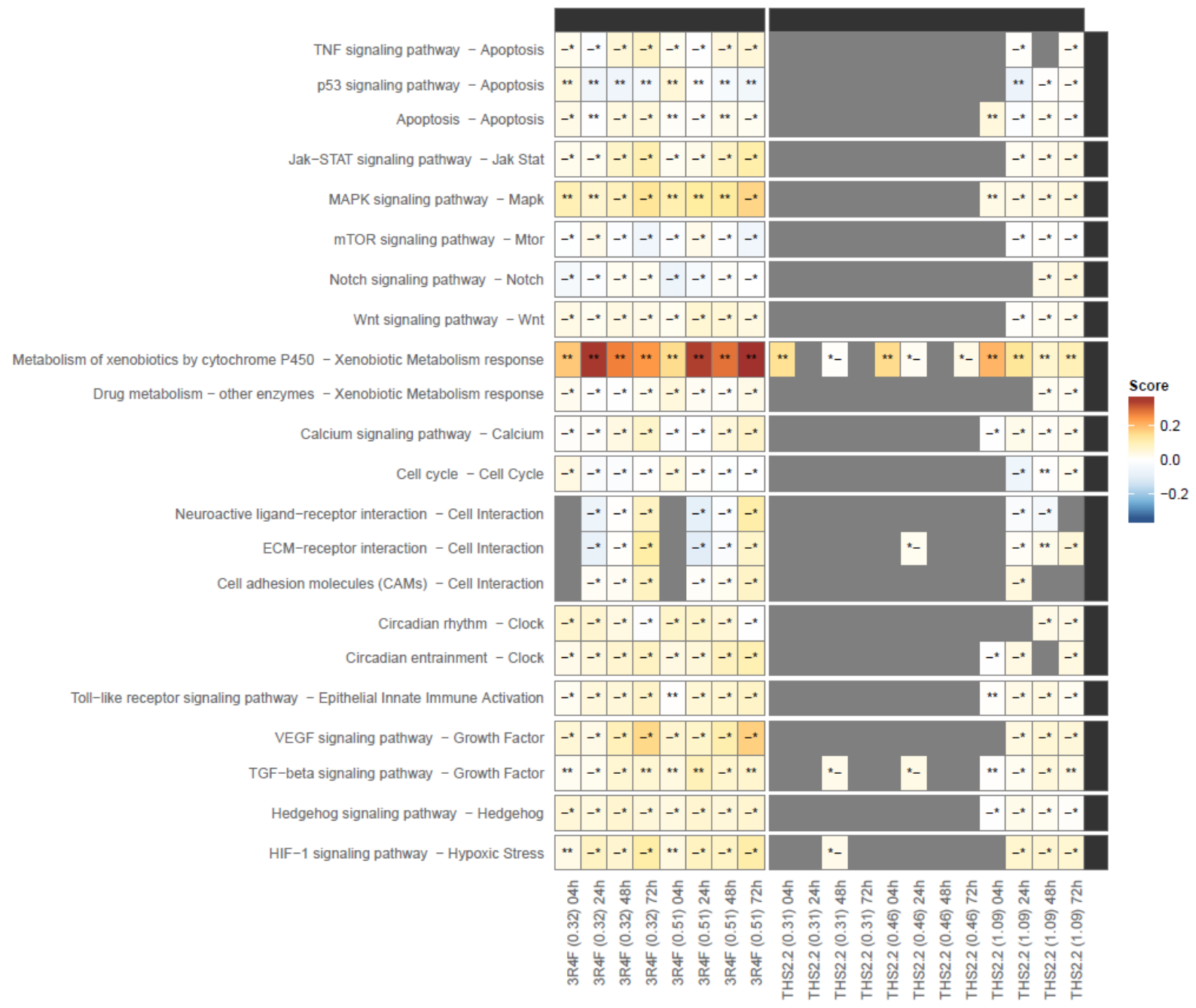




\section{Figure S8}

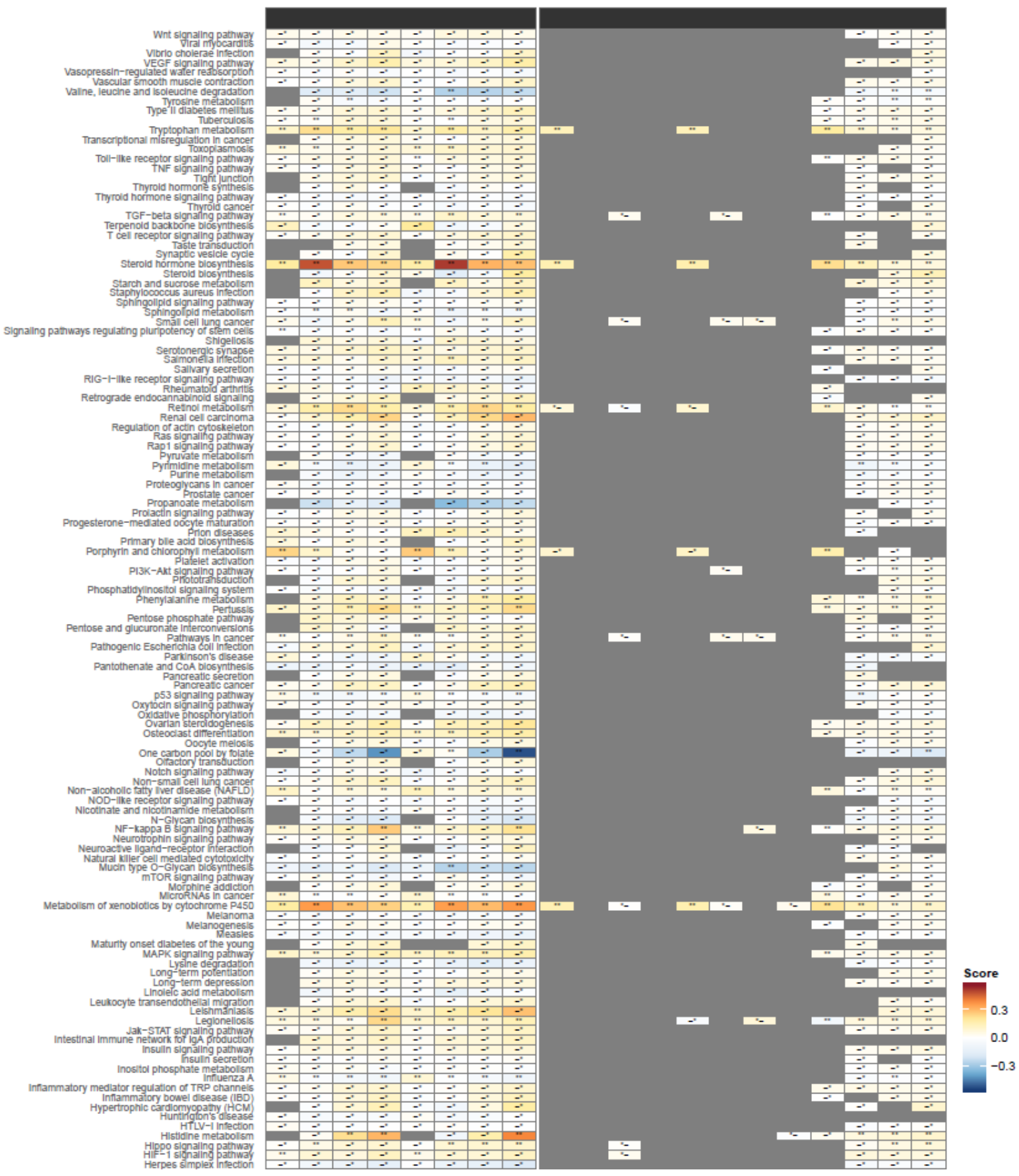




\section{Figure S8 (continues)}

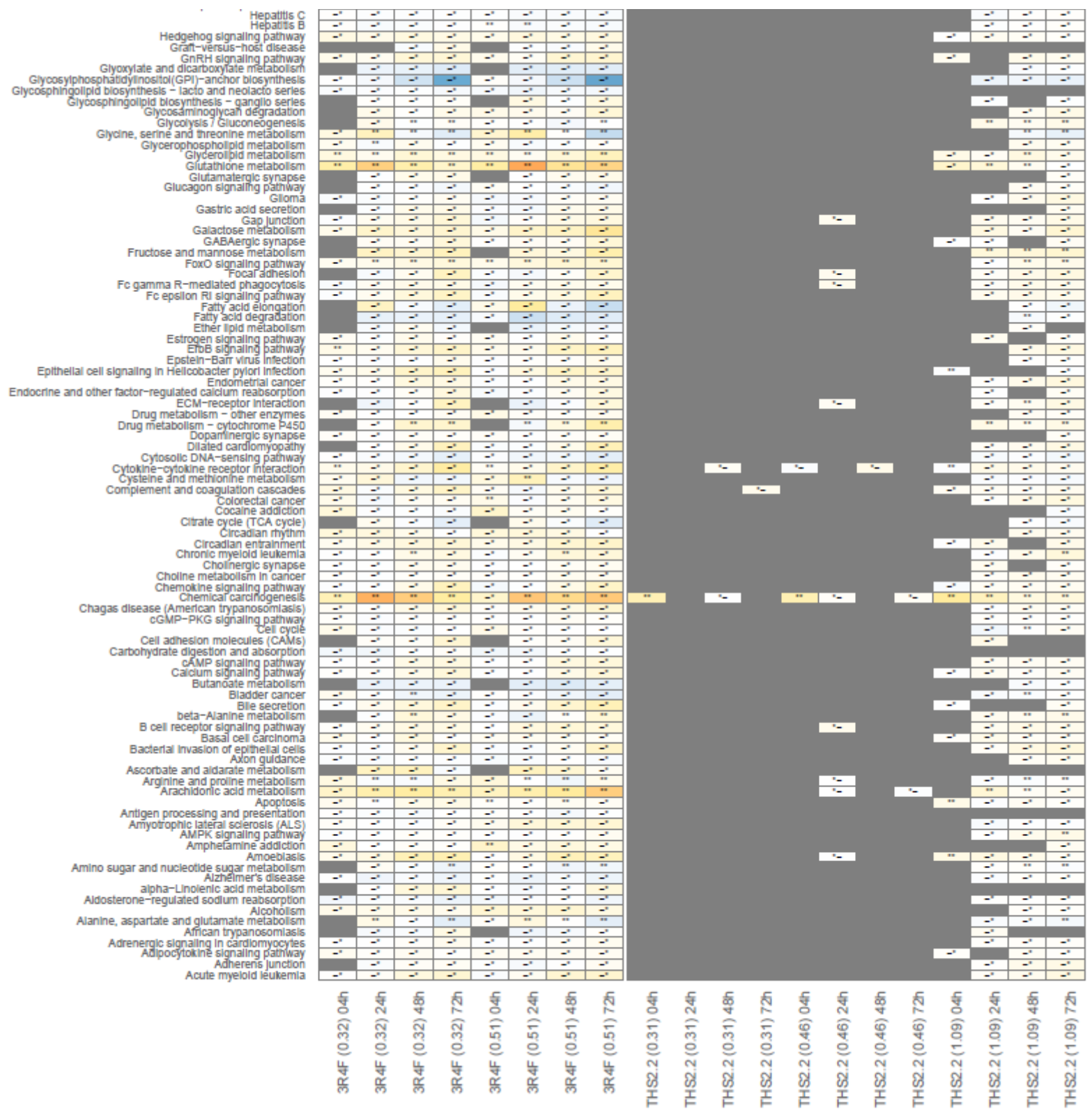




\section{Figure S9}

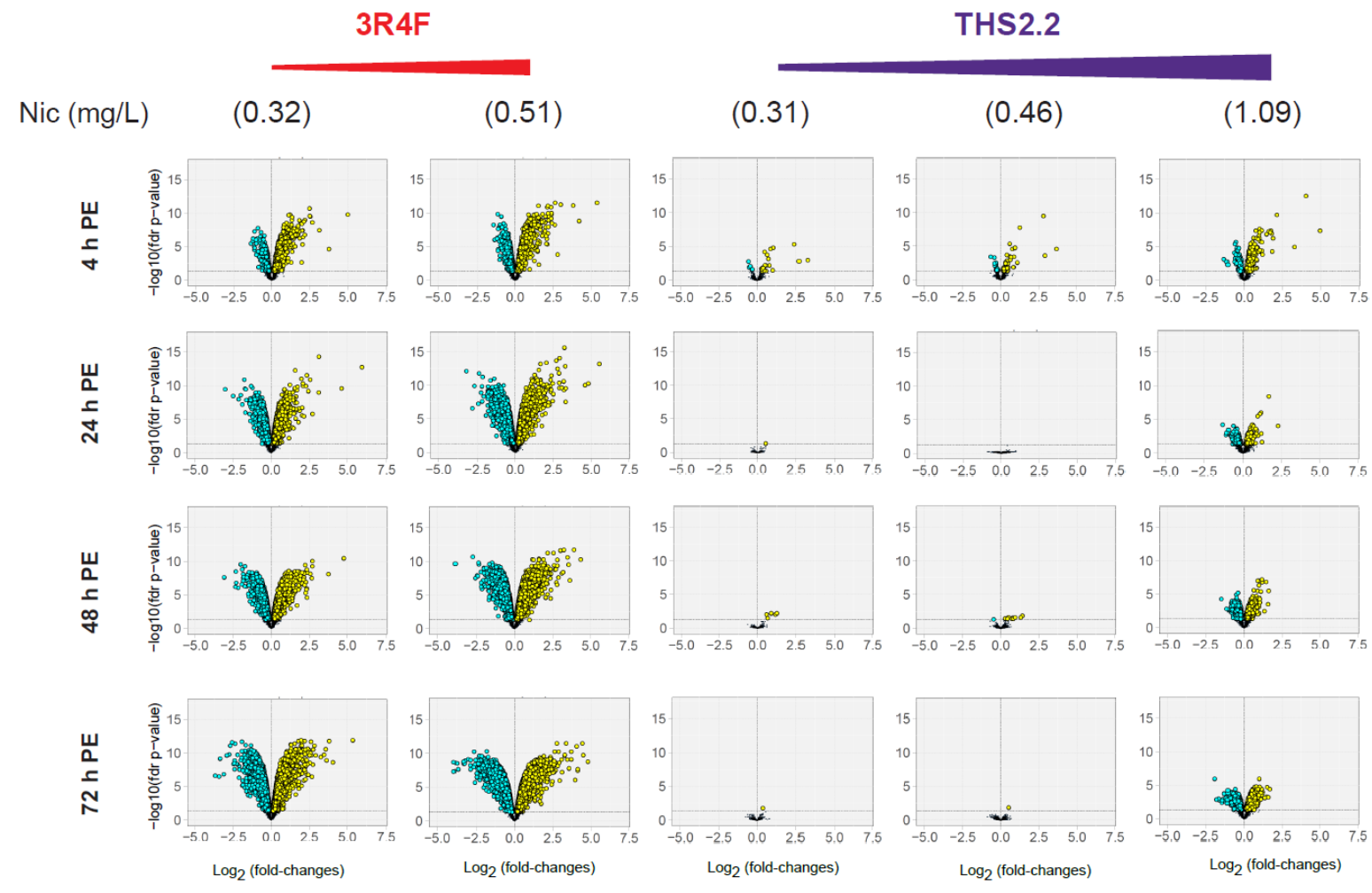




\section{Figure S10}

A

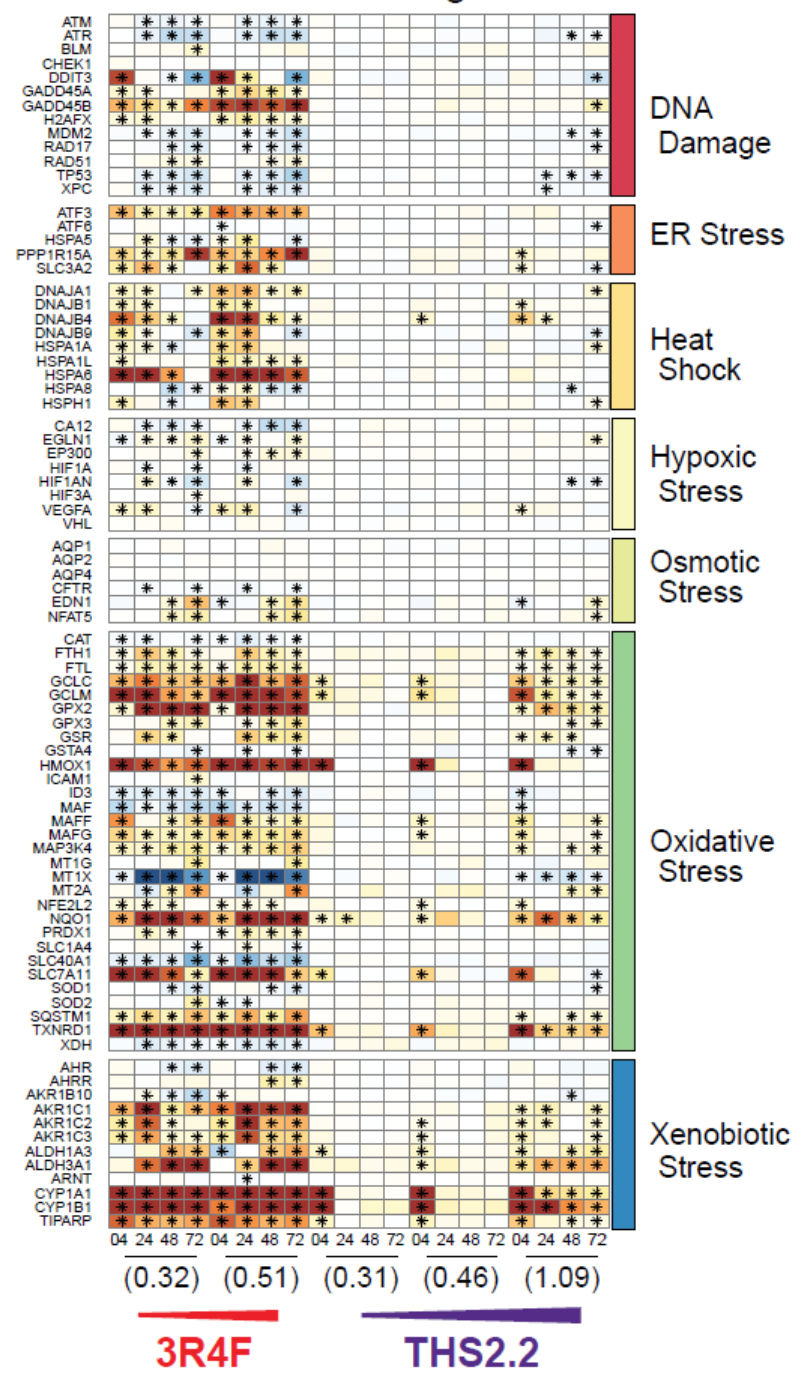

B
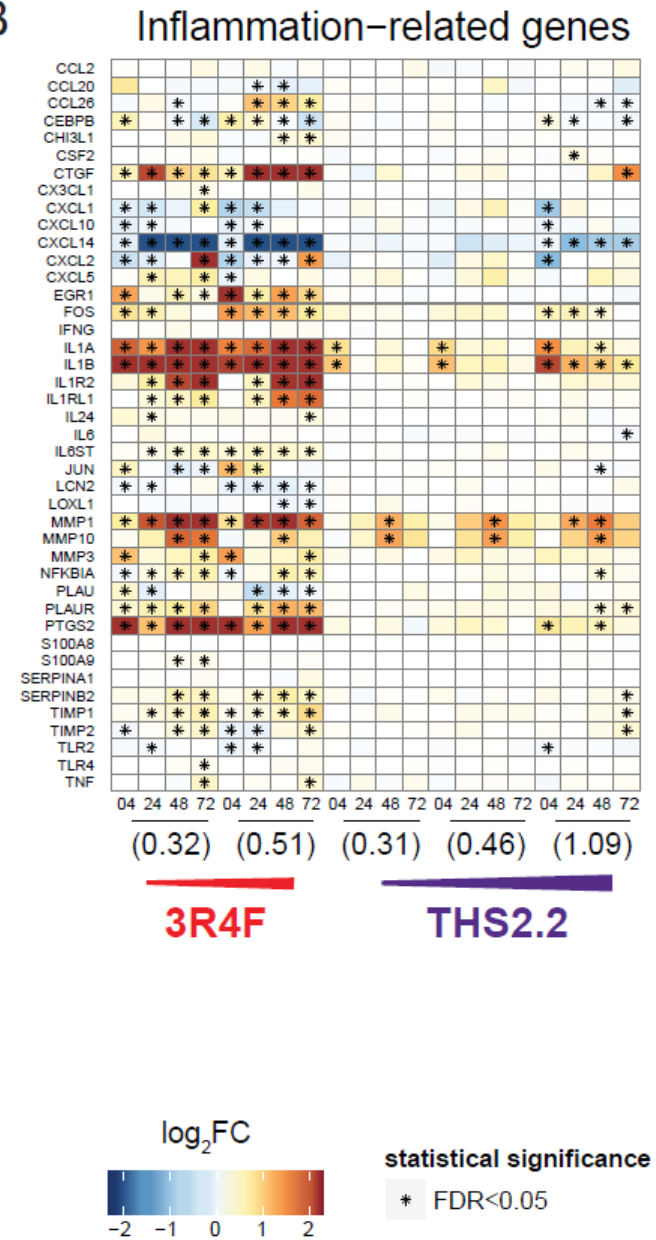


\section{Figure S11}

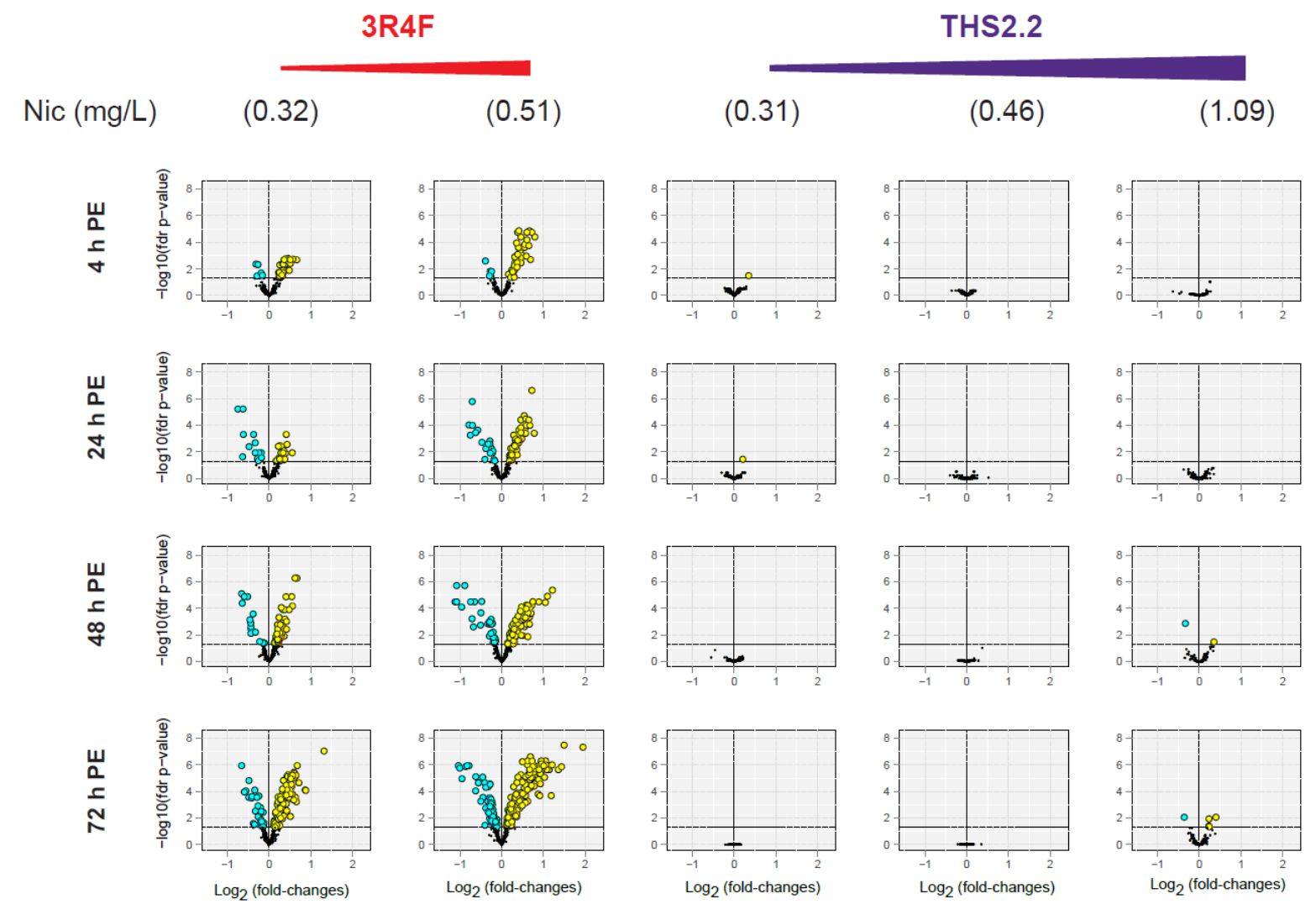




\section{Figure S12}

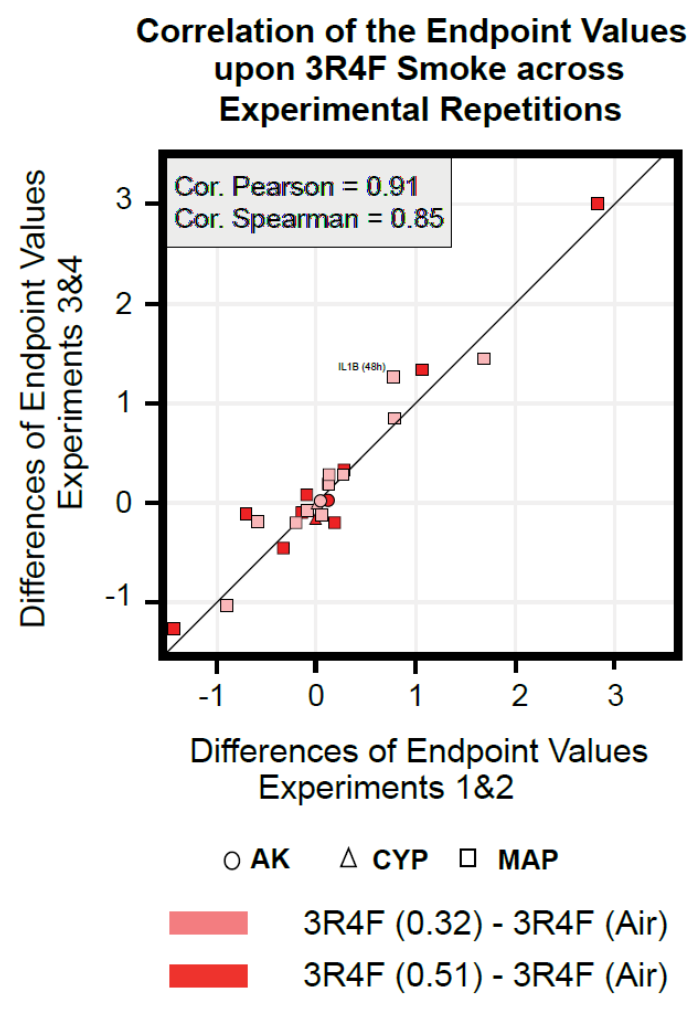

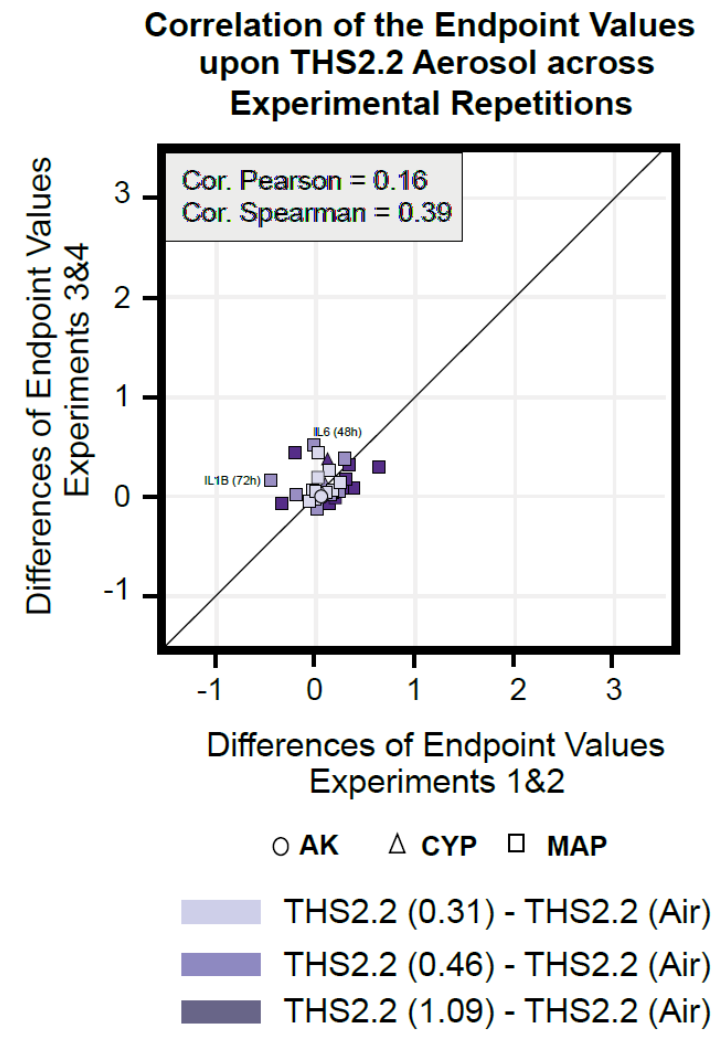




\section{Figure S13}

A

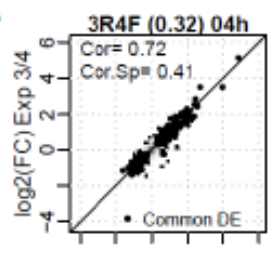

3R4F (0.32) 24h
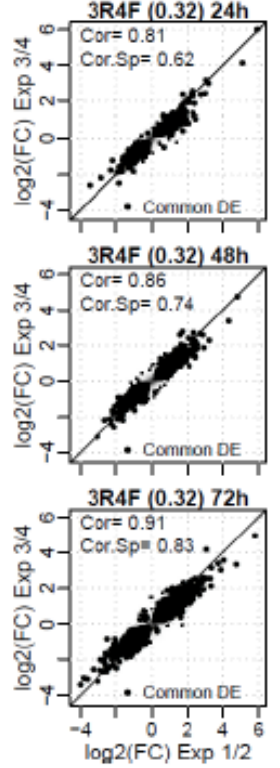

B

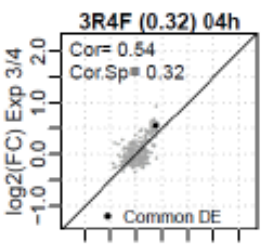

$3 R 4 F(0.32) 24 \mathrm{~h}$

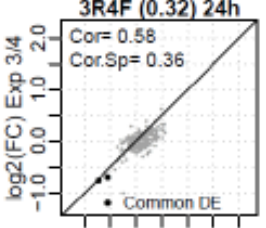

$3 R 4 F(0.32) 48 \mathrm{~h}$

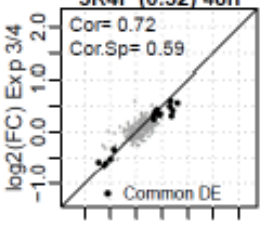

3R4F $(0.32) 72 \mathrm{~h}$

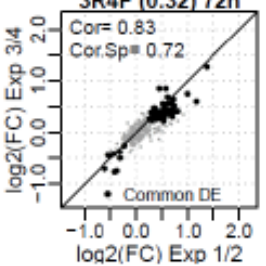

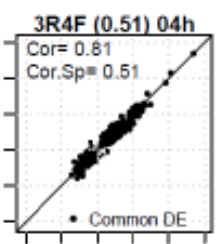

3R4F (0.51) 24h

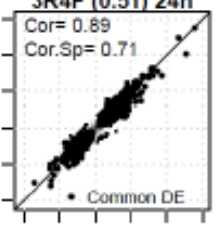

3R4F $(0.51) 48 \mathrm{~h}$

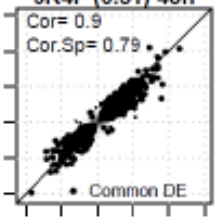

3R4F $(0.51) 72 \mathrm{~h}$

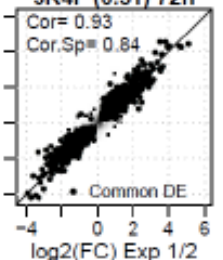

log $2(F C) \operatorname{Exp} 1 / 2$

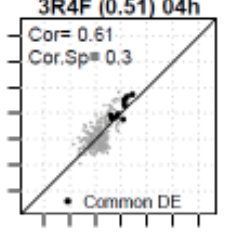

3R4F (0.51) 24h

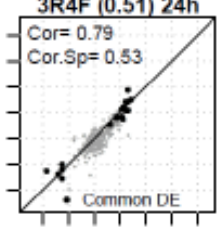

$3 R 4 F(0.51) 48 \mathrm{~h}$
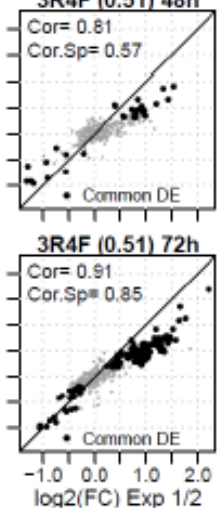
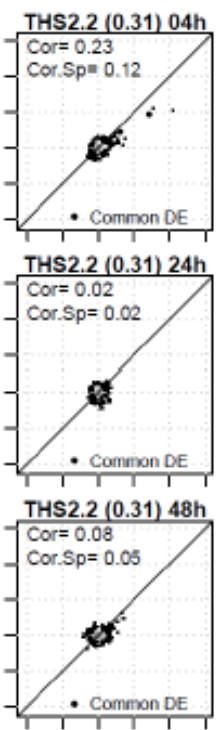

THS2.2 $(0.31) 72 \mathrm{~h}$

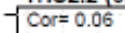

Cor.Sp= 0.02

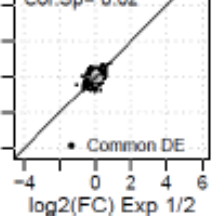

THS2.2 (0.31) 04h
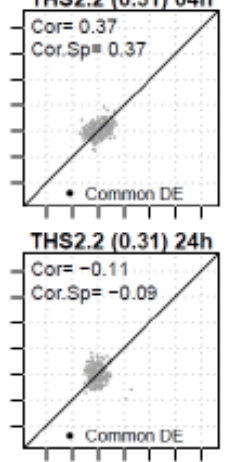

THS2.2 (0.31) $48 \mathrm{~h}$

Cor $=0.32$

Cor. $. \mathrm{sp}=0.23$
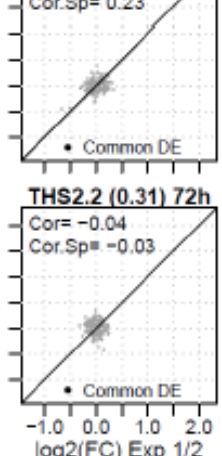
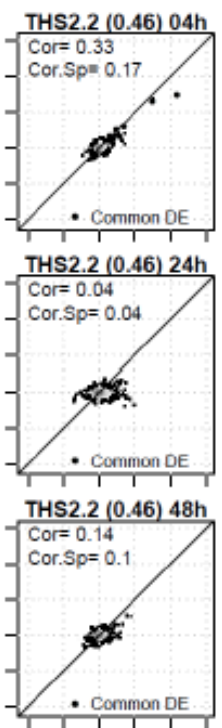

THS2.2 (0.46) 72h

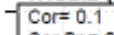

Cor.Sp= 0.05

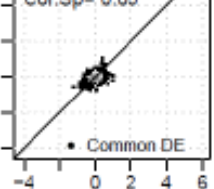

$\log 2$ (FC) Exp 1/2

THS2.2 (0.46) 04h
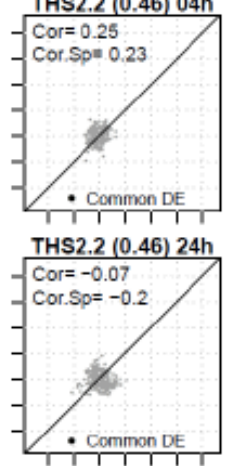

THS2.2 (0.46) 48h
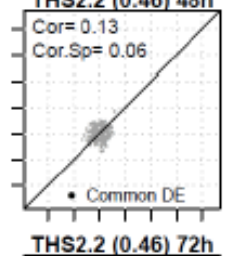

Cor $=-0.05$

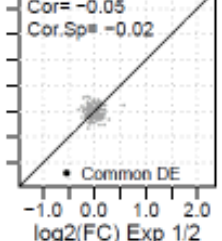

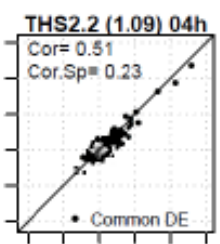
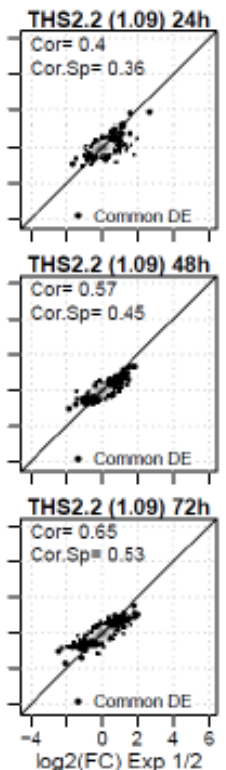

THS2.2 (1.09) 04h

- Cor $=0.3$

Cor.Sp= 0.19
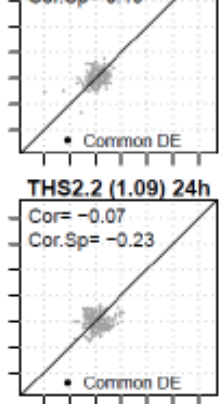

THS2.2 (1.09) 48h

Cor $=0.37$

Cor. $. p=0.17$

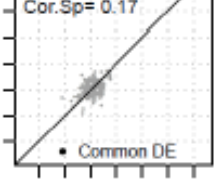

THS2.2 (1.09) 72h

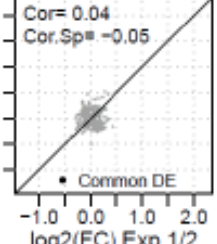

